\title{
Review \\ Quantum Current Algebra Symmetry and Description of Boltzmann Type Kinetic Equations in Statistical Physics
}

\author{
Lev I. Ivankiv ${ }^{1, \dagger}$, Yarema A. Prykarpatsky ${ }^{2}$ (]) , Valeriy H. Samoilenko ${ }^{3}$ and Anatolij K. Prykarpatski ${ }^{4, *}$ \\ 1 Department of Electronics and Computer Technologies, Lviv State University, 79-001 Lviv, Ukraine; \\ pryk.anat@gmail.com \\ 2 The Department of Applied Mathematics, University of Agriculture in Krakow, Balicka 253c, \\ 30-198 Krakow, Poland; yarpry@gmail.com \\ 3 Department of Mathematical Physics, Taras Shevchenko National University of Kyiv, 01-601 Kyiv, Ukraine; \\ valsamyul@gmail.com \\ 4 Department of Computer Science and Teleautomatics, Cracov University of Technology, \\ 31-155 Cracow, Poland \\ * Correspondence: pryk.anat@cybergal.com \\ + Dedicated to the bright memory of our friend and teacher-The talented ukrainian physicist Lev I. Ivankiv \\ with admiration.
}

check for

updates

Citation: Ivankiv, L.I.; Prykarpatsky, Y.A.; Samoilenko, V.H.; Prykarpatski, A.K. Quantum Current Algebra Symmetry and Description of Boltzmann Type Kinetic Equations in Statistical Physics. Symmetry 2021, 13, 1452. https://doi.org/10.3390/ sym 13081452

Academic Editors: Sayantan Choudhury and Savan Kharel

Received: 26 May 2021

Accepted: 9 July 2021

Published: 9 August 2021

Publisher's Note: MDPI stays neutral with regard to jurisdictional claims in published maps and institutional affiliations.

Copyright: (C) 2021 by the authors Licensee MDPI, Basel, Switzerland. This article is an open access article distributed under the terms and conditions of the Creative Commons Attribution (CC BY) license (https:// creativecommons.org/licenses/by/ $4.0 /)$.

\begin{abstract}
We review a non-relativistic current algebra symmetry approach to constructing the Bogolubov generating functional of many-particle distribution functions and apply it to description of invariantly reduced Hamiltonian systems of the Boltzmann type kinetic equations, related to naturally imposed constraints on many-particle correlation functions. As an interesting example of deriving Vlasov type kinetic equations, we considered a quantum-mechanical model of spinless particles with delta-type interaction, having applications for describing so called Benney-type hydrodynamical praticle flows. We also review new results on a special class of dynamical systems of BoltzmannBogolubov and Boltzmann-Vlasov type on infinite dimensional functional manifolds modeling kinetic processes in many-particle media. Based on algebraic properties of the canonical quantum symmetry current algebra and its functional representations, we succeeded in dual analysis of the infinite Bogolubov hierarchy of many-particle distribution functions and their Hamiltonian structure. Moreover, we proposed a new approach to invariant reduction of the Bogolubov hierarchy on a suitably chosen correlation function constraint and deduction of the related modified BoltzmannBogolubov kinetic equations on a finite set of multi-particle distribution functions. There are also presented results of application of devised methods to describing kinetic properties of a many-particle system with an adsorbent surface, in particular, the corresponding kinetic equation for the occupation density distribution function is derived.
\end{abstract}

Keywords: kinetic equations; Bogolubov chain; multi-particle distribution function; current algebra; Wigner representation; functional equations; reduction theory; Lie-Poisson brackets; invariant functional submanifolds; surface adsorption centers

PACS: $11.10 . E f ; 11.15 . K c ; 11.10 .-z ; 11.15 .-q ; 11.10 . W x ; 05.30 .-d$

\section{Introduction}

As it is well known [1-8], the objective of the kinetic theory of many-particle dynamical systems is to explain the properties of macroscopic non-equilibrium state of gases (or liquids) in terms of microscopic properties of individual gas molecules and interaction forces between them. The main task of this theory is to provide microscopic explanation of the fact that a great variety of many-particle flows may be described with NavierStokes hydrodynamical equations, as well as to find analytical expressions for viscosity and thermal conductivity coefficients in terms of microscopic properties of molecules 
and other characteristics. Kinetic theory of gases takes origin in classic works of Daniel Bernoulli, Rudolf Clausius, and especially of James Clerk Maxwell, who was the first to apply statistical methods to gas properties calculations, having realized that random gas molecules movement may be described by means of distribution functions. Apart from the distribution form, Maxwell has also found the transport equation for mass. He has found "microscopic" expressions for transport coefficients for gas constituted by the so-called Maxwell molecules, which interact through push forces, which are inversely proportional to the interparticle distance in the fifth power. He has also shown that the viscosity and thermal conductivity coefficients do not depend on the gas density. Another significant contribution to the statistical mechanics theory was made by Boltzmann, who obtained his world-known equation for time-dependent distribution function for dilute gas in nonequilibrium state in 1872. The Boltzmann equation provides a microscopic description of non-equilibrium processes in dilute gases as well as of gas transition to equilibrium state. Making use of the Boltzmann equation, Chapman and Enskog [9] derived the NavierStokes equation and obtained the expressions for dilute gas particles transport coefficients, which are characterized by pair interaction through short interaction forces. Even now, more than hundred years after Boltzmann derived his equation, the kinetic theory for dilute gases mainly studies stationary solutions to this equation for various initial and boundary conditions and different gas components. Fundamental results of the consistent derivation of the Boltzmann equation from the Liouville equation were obtained by Bogolubov in 1946 and later by Cohen, Green, Kirkwood, and Yvon [1,3-6]. The approach to the Boltzmann equation through the Liouville equation was found to be important for two reasons. First, it allowed to replace the Stosszahlansatz hypothesis of Boltzmann with more fundamental principle of the attenuation of the Bogolubov weakening correlations [2], and second, it also allowed to generalize the Boltzmann equation to dense gases. In the fundamental work of Bogolubov [2] there was also suggested and developed a new method for the study of the distribution function for gas, the so called method of generating functionals. By means of the generating functional there is taken down in the compact form the entire infinite hierarchy of the non-equilibrium equations for the distribution functions (Bogolubov's hierarchy or BBGKY [1,6]), which under certain limitations on the functional allows for their various partial as well as approximate solutions. Solid explanation of the properties as well as possibilities of the method was later provided in the works of Bogolubov M.M. (Jr.) and co-workers [7,10-12] drawing upon the introduction of the so called quantum generating functional of the Bogolubov type and the study of its algebraic properties. Namely, it was found that the hierarchy of the Bogolubov equations in terms of the quantum generating functional realizes irreducible unitary representations of the quantum current Lie algebra $G=\operatorname{Diff}(\Lambda) \ltimes \mathcal{J}(\Lambda ; \mathbb{R})$, which is a semi-direct product of the group of diffeomorphisms of a domain $\Lambda \subset \mathbb{R}^{3}$ and the space of Schwartz functions on it. Within this approach all classic solutions are obtained, respectively, after moving to Wigner representations $[3,10,13,14]$ for basic operators of the initial algebraic structure. For further analysis of the Bogolubov functional equation and the respective variety of its solutions for the given many-particle dynamical system, we are going to provide below its consistent derivation within the framework of the algebraic Bogolubov approach (Bogolubov M.M. Jr.) and establish its Hamiltonianity with respect to the special Lie-Poisson bracket on the orbits of the co-adjoined representation of the Wigner Lie algebra of quantum operators of the obeservable quanitites. We study in detail a special class of dynamical systems of Boltzmann-Bogolubov and Boltzmann-Vlasov type on infinite dimensional functional manifolds modeling kinetic processes in many-particle media. We analyzed an interesting example of Vlasov type kinetic equations generated by a quantum-mechanical model of spinless particles with delta-type interaction, having applications for describing so called Benney-type hydrodynamical particle flows. Based on the algebraic properties of the canonical quantum symmetry current algebra and its functional representations, devised before in [15], we succeeded in dual analysis of the infinite Bogolubov hierarchy of many-particle distribution functions and their Hamiltonian structure. Moreover, we 
proposed a new approach to invariant reduction of the Bogolubov hierarchy on a suitably chosen invariant subspace and deduced the related modified Boltzmann-Bogolubov kinetic equations on a finite set of multi-particle distribution functions. There are also presented results of application of devised methods to describing kinetic properties of a many-particle system with an adsorbent surface, in particular, there is derived the corresponding kinetic equation for the occupation density distribution function.

\section{Kinetic Equations: Introductory Quantum-Mechanical Example}

\subsection{The Quantum Many-Particle Hamiltonian and the Related Quasi-Classical Averaging}

It is well known [16,17] that the classical Boltzmann equation under no correlation condition describes long waves in a dense gas with a short-range interaction potential. The same equation, which is called the Vlasov equation [18], is equivalent to the hydrodynamic equations for long waves in an ideal incompressible liquid with a free surface under gravity. It also quite easy to see that this equation in the classical "random phase" approximation reduces to the well-known nonlinear model in plasma physics. These equivalences for the kinetic and hydrodynamic equations can be used for studying dynamical chaotic behavior in many-particle systems and often accompanying it turbulence arising in both in gas and fluid flows. These aspects are very important as dynamical many-particle systems possess an important intrinsic property of particle motion in a liquid-convective mass transfer of particles in a fixed volume-which is known to always accompany a transition from laminar to turbulent flow and cause convective vortex wise motion. It is also important that the realistic models to possess an intrinsic so-called "dry" viscosity for the particle flows.

To take into account these phenomena, we will introduce a new generalized dynamical system for the flow of particles in a domain $\Lambda \subset \mathbb{R}^{3}$, namely a Boltzmann-Bogolubov kinetic equation in the Vlasov approximation with no many-particle correlation, describing long waves in a dense gas of particles with a short-range interaction potential. We will also derive the associated Benney type system of spatial equations containing the convective terms in a form that is especially convenient for describing the turbulence phenomenon. Moreover, the mathematical model of interacting particles we analyzed is such that the associated Benney type system of equations appears to be Hamiltonian and reduces to an interesting related three-dimensional hydrodynamic type particle flow.

Let us consider a quantum dynamical system on $\Lambda \subset \mathbb{R}^{3}$ consisting of $N \in \mathbb{N}$ identical spinless particles with the singular Hamiltonian operator

$$
\hat{H}=-\frac{\hbar^{2}}{2 m} \sum_{j=1}^{N}\left\langle\nabla_{x_{j}} \mid \nabla_{x_{j}}\right\rangle+\frac{1}{2} \sum_{j \neq k}^{N} \delta\left(x_{j}-x_{k}\right)\left[\beta+\left\langle\alpha \mid \frac{\hbar}{i}\left(\nabla_{x_{j}}+\nabla_{x_{k}}\right)\right\rangle\right],
$$

where $\alpha \in \mathbb{E}^{3}$ is an external constant momentum vector and $\beta \in \mathbb{R}_{+}$is a real parameter, $\hbar$ is the Planck's constant (divided by $2 \pi$ ), $m \in \mathbb{R}_{+}$is the particle mass, and $\delta(x-y), x, y \in \Lambda$ is the classical Dirac delta-function. Then, corresponding Wigner's transformation [3] to a quasiclassical weak limit as $\hbar \rightarrow 0$ yields $\lim _{\hbar \rightarrow 0} \hat{H} \rightarrow H$, where the classical Hamiltonian function $H: T^{*}(\Lambda)^{N} \rightarrow \mathbb{R}$ has the form

$$
H=\frac{1}{2 m} \sum_{j=1}^{N} p_{j}^{2}+\frac{1}{2} \sum_{j \neq k}^{N} \delta\left(x_{j}-x_{k}\right)\left[\beta+\left\langle\alpha \mid\left(p_{j}+p_{k}\right)\right\rangle\right] .
$$

Here, the $x_{j} \in \Lambda, j=\overline{1, N}$, are the coordinates of the particles on the axis $\mathbb{R}$. The Heisenberg operator commutator $[\cdot, \cdot]$ for evolution of dynamical observables [3] becomes the standard canonical Hamiltonian bracket $\{\cdot, \cdot\}$, viz.

$$
[\cdot, \cdot] \frac{i}{\hbar} \stackrel{\hbar \rightarrow 0}{\longrightarrow}\{\cdot, \cdot\},
$$


in accordance with the classical Born principle. Therefore, on the cotangent phase space $T^{*}(\Lambda)^{N}$ the Hamiltonian equations take the following form:

$$
d x_{j} / d t=\left\{H, x_{j}\right\}=\partial H / \partial p_{j}, \quad d p_{j} / d t=\left\{H, p_{j}\right\}=-\partial H / \partial x_{j},
$$

where $t \in \mathbb{R}$ is an evolution parameter and $\left(x_{j}, p_{j}\right) \in T^{*}(\Lambda), j=\overline{1, N}$.

In view of the singularities in (2), the Equation (4) are in general not solvable for arbitrary Cauchy data and large $N \in \mathbb{N}$. Therefore, because of our "kinetic" interest in the motion of (4), we pass further to its statistical description $[3,19,20]$, using the BoltzmannBogolubov distribution function $F: T^{*}(\Lambda) \times \mathbb{R} \rightarrow D^{\prime}\left(T^{*}(\Lambda)\right)$, defined by

$$
F(x, p ; t):=\sum_{j=1}^{N} \delta\left(x-x_{j}(t)\right) \delta\left(p-p_{j}(t)\right) .
$$

Here, $(x, p) \in T^{*}(\Lambda)$ and $\left(x_{j}(t), p_{j}(t)\right) \in T^{*}(\Lambda), j=\overline{1, N}$, is a solution of the Hamiltonian Equation (4). It is well known [3] that the distribution function (5) satisfies the standard Liouville-Hamilton equation

$$
d F / d t=\{F, H\}
$$

Now, we apply the averaging operator $\langle\cdot\rangle$ to the distribution function (5) assuming no many-particle correlation over all initial states of (6). The averaging operation on (6) results in the kinetic Boltzmann-Vlasov equation $[3,19]$ of the form

$$
d f / d t=<\{F, H\}>:=\{\{F, H\}\},
$$

where $f=f(x, p ; t):=\left\langle F(x, p ; t)>,(x, p ; t) \in T^{*}(\Lambda) \times \mathbb{R}_{+}\right.$, is the statistically averaged distribution function (5) and $\{\{\cdot, \cdot\}\}$ is a new "averaged" Poisson bracket on the infinitedimensional functional space $M_{(f)} \subset C^{\infty}\left(T^{*}(\Lambda), \mathbb{R}_{+}\right)$, which for a pair of functionals $\gamma, \mu \in D\left(M_{(f)}\right)$ has the form [10,17]:

$$
\{\{\gamma, \mu\}\}=\int_{\Lambda} d x \int_{\mathbb{R}^{3}} d p f(x, p ; t)\{\operatorname{grad} \gamma, \operatorname{grad} \mu\}(x, p ; t)
$$

and is called the canonical Lie-Poisson bracket $[10,17,21,22]$. The Hamiltonian $H \in D\left(M_{(f)}\right)$ in (7) is given by

$$
\begin{aligned}
H= & \int_{\Lambda} d x\left[\int_{\mathbb{R}^{3}} d p \frac{p^{2}}{2 m} f(x, p ; t)+\frac{\beta}{2}\left(\int_{\mathbb{R}^{3}} d p f(x, p ; t)\right)^{2}+\right. \\
& \left.+\int_{\mathbb{R}^{3}} d p f(x, p ; t) \int_{\mathbb{R}^{3}} d q\langle\alpha \mid q\rangle f(x, q ; t)\right]
\end{aligned}
$$

and "grad" denotes here the standard Euler variational derivative on $D\left(M_{(f)}\right)$.

To derive (8) let us consider the phase space $M_{(f)}=T^{*}(\Lambda)$ endowed with the canonical Poisson bracket $\{f, g\}=\left\langle\nabla_{p} f \mid \nabla_{x} g\right\rangle-\left\langle\nabla_{p} g \mid \nabla_{x} f\right\rangle$, where $f, g \in D\left(M_{(f)}\right)$ are smooth functions on $M_{(f)}$. The space $D\left(M_{(f)}\right)$ has the structure of a Lie algebra $\mathcal{G} \simeq$ $\left(\mathcal{D}\left(M_{(f)}\right) ;\{\cdot, \cdot\}\right)$ with respect to this bracket (8).

Let $\mathcal{G}^{*}$ be the adjoint or dual of $\mathcal{G}$, i.e., the space of continuous linear functionals on $\mathcal{G}$. The space $\mathcal{G}$ is a pre-Hilbert space with respect to the scalar product defined by

$$
(f \mid g):=\int_{\Lambda} d x \int_{\mathbb{R}^{3}} d p f(x, p) g(x, p) .
$$


Moreover, the adjoint sdpace $\mathcal{G}^{*} \simeq \mathcal{G}$ is a Hilbert space, on which the above scalar product is invariant with respect to the Poisson bracket $\{\cdot, \cdot\}$ in the sense that

$$
(f \mid\{g, h\})=(\{f, g\} \mid h)
$$

for all $f, g, h \in \mathcal{G}$. This structure enables us to determine the map grad $: D\left(\mathcal{G}^{*}\right) \rightarrow \mathcal{G}$ by means of the formula $(g \mid \operatorname{grad} \gamma(f))=\left.\frac{d}{d \varepsilon} \gamma(f+\varepsilon g)\right|_{\varepsilon=0}$ for arbitrary $f, g \in \mathcal{G}^{*} \simeq \mathcal{G}$. Consequently, grad $\gamma(f) \in \mathcal{G}$ is here completely equivalent to the variational Euler derivative on the space of local functionals $\gamma \in D\left(\mathcal{G}^{*}\right)$ at point $f \in \mathcal{G}^{*} \simeq \mathcal{G}$.

The canonical Hamiltonian structure $\{\{\cdot, \cdot\}\}$ on the manifold $\mathcal{G}^{*}$ can now be expressed via the well-known Lie-Poisson formula $[10,17,21-23]$

$$
\{\{\gamma, \mu\}\}=(f \mid\{\operatorname{grad} \gamma(f), \operatorname{grad} \mu(f)\})
$$

which, obviously, coincides with (8). To reveal the essence of the formula (7) we consider a coadjoint action of the Lie algebra $\mathcal{G}$ on $\mathcal{G}^{*}$, suitably generating a flow $d f / d t=-a d_{\operatorname{grad} \gamma(f)}^{*} f$, where $t \in \mathbb{R}$ is a real evolution parameter and grad $\gamma(f) \in \mathcal{G}$ is the flow generator at $f \in \mathcal{G}^{*}$. Then, owing to the invariance of the scalar product on $\mathcal{G}$, the above vector field is equivalent to the following commutator representation on $\mathcal{G}: d f / d t=\{f$, grad $\gamma(f)\}$, which in turn is equivalent to (8) after an identification $\gamma=H \in D\left(M_{(f)}\right) \subset D\left(\mathcal{G}^{*}\right)$.

One easily observes that the Hamiltonian function $H$ given by (9) is a conservation law for the Boltzmann-Vlasov system (7), i.e., $d H / d t=0$ for all $t \in \mathbb{R}$. Apart from this conservation law, the dynamical system (7) possesses the following additional invariant functionals on $\mathcal{G}^{*} \cong \mathcal{G}$ :

$$
N=\int_{\Lambda} d x \int_{\mathbb{R}^{3}} d p f(x, p ; t), \quad P=\int_{\Lambda} d x \int_{\mathbb{R}^{3}} d p p f(x, p ; t),
$$

where $N$ is the number of particles and $P \in D\left(M_{(f)}\right)$ is the total particle momentum of the system.

\subsection{Boltzmann Equation, the Associated Momentum Problem and Related Hydrodynamic Type Particle Flow}

Below, we shall show that the Boltzmann-Vlasov system (7) with Hamiltonian (9) can be represented in the equivalent commutator form

$$
d f / d t=\{f, \operatorname{grad} H(f)\},
$$

where $f \in D\left(M_{(f)}\right)=\mathcal{G}^{*} \simeq \mathcal{G}$, and possesses (with respect to the Lie-Poisson bracket) additional conservation laws, important for analysis its exact solutions [7,24].

The exact form of the Boltzmann-Vlasov Equation (11) is

$$
f_{t}=-\left\langle p \mid \nabla_{x} f\right\rangle-\left\langle\alpha \mid \nabla_{x}\left(a_{0} f\right)\right\rangle+\left\langle\nabla_{x}\left|\left(\beta a_{0}+a_{1}\right)\right| \nabla_{p} f\right\rangle+\left\langle\nabla_{x} a_{0}\right| \nabla_{p}(\langle\alpha \mid p\rangle f),
$$

where the momentum functionals $a_{n} \in M_{\left(\mathbb{Z}_{+}\right)}, n \in \mathbb{Z}_{+}$, are defined as follows:

$$
a_{n}(x, t):=\int_{\Lambda} d x\langle\alpha \mid p\rangle^{n} f(x, p ; t)
$$

for all $x \in \Lambda$ and $t \in \mathbb{R}_{+}$. In momentum terms (13), the Hamiltonian (9) as a functional $H \in D\left(M_{\left(\mathbb{Z}_{+}\right)}\right)$takes the form $\backslash$ with the Lie-Poisson bracket (8) on the manifold $M_{\left(\mathbb{Z}_{+}\right)}$ being given by the expression $[16,17,23,25]$ :

$$
\{\gamma, \mu\}_{\mathcal{L}}:=\int_{\Lambda} d x\langle\operatorname{grad} \gamma, \theta(a) \operatorname{grad} \mu\rangle
$$


where $\theta(a):=\left[m a_{m+n-1} \nabla_{x}+n \nabla_{x} a_{m+n-1}\right], m, n \in \mathbb{Z}_{+}$, is a skew-symmetric matrix operator on $M_{\left(\mathbb{Z}_{+}\right)}, \operatorname{grad}(\ldots)=\left(\delta / \delta a_{n}(\ldots)\right)^{\top}, n \in \mathbb{Z}_{+}$, and $\langle\cdot \mid \cdot\rangle$ is the standard inner product on the space $l_{2}(\Lambda ; \mathbb{R})$ of real square-summable sequences.

The dynamical system for the momentum functions corresponding to (12) is

$$
d a_{n} / d t=\left\{H, a_{n}\right\}_{\theta}=-a_{n+1, x}-n a_{n}\left\langle\alpha \mid \nabla_{x} a_{0}\right\rangle-n a_{n-1}\left\langle\nabla_{x}\left(\beta a_{0}+\alpha a_{1}\right) \mid \nabla_{x}\left(a_{0} a_{n}\right)\right\rangle,
$$

where $n \in \mathbb{Z}_{+}$and $\alpha \in \mathbb{E}^{3}, \beta \in \mathbb{R}$ are some constant parameters. The Equations (15) are called the generalized Benney type momentum system. In particular, for $n=0$ and $n=1$ we obtain the following from (15):

$$
\begin{aligned}
& d a_{0} / d t=-\left\langle\alpha \mid \nabla_{x}\left(a_{0}^{2}\right)\right\rangle \\
& d a_{1} / d t=-a_{2, x}-2\left\langle\alpha \mid \nabla_{x}\left(a_{0} a_{1}\right)\right\rangle-\beta a_{0}\left\langle\alpha \mid \nabla_{x} a_{0}\right\rangle .
\end{aligned}
$$

It is interesting to remark here that the simplest substitution $f(x, p ; t)=\rho(x, t) \delta(p-$ $u(x, t)),(x, p) \in T^{*}(\Lambda)$, in (16), responsible for the hydrodynamic representation of the averaged distribution function (6), we obtain the following new nonlinear Benney type system of equations:

$$
K[u, \rho]=\left\{\begin{array}{l}
u_{t}=-\left\langle u \mid \nabla_{x}\right\rangle u-\nabla_{x}(\beta \rho+2\langle\alpha \mid u\rangle \rho) \\
\rho_{t}=-\langle\nabla \mid(u \rho)\rangle-2\left\langle\alpha \rho \mid \nabla_{x} \rho\right\rangle
\end{array}\right.
$$

where $(u, \rho)^{\top} \in M_{(u, \rho)} \subset C^{\infty}\left(\Lambda, \mathbb{R}^{3} \times \mathbb{R}\right)$ are particle velocity and density functions, owing to the natural physical identifications of our particle system as a suitable hydrodynamical flow in $\Lambda$.

Below we develop the classical Bogolubov approach to deriving kinetic equations for many-particle system, based on the fundamental quantum current algebra symmetry and its functional representations. The latter makes it possible to present a new description of the infinite hierarchy of distribution functions and construct the corresponding correctly decoupled infinite systems of kinetic equations for distribution functions.

The first draft [15] of this review was initiated and written jointly with our colleague and talanetd university teacher Prof. Lev I. Ivankiv, who recently passed away. The further work on the review was actively continued jointly with Prof. Valeriy H. Samoilenko and Dr. Yarema A. Prykarpatsky, whose impact in developing the Lev I. Ivankiv's ideas appeared both essential and decisive while preparing a final version of the manuscript.

\section{The Bogolubov Generating Functional in Non-Equilibrium Statistical Mechanics and Its Wigner Quasi-Classical Representation}

3.1. The Current Lie Algebra Representation

Let us be given the Hilbert space $\Phi$ of the states in the non-equilibrium quantum mechanics. There are defined field annihilation operator $\psi^{+}(x)$ and creation operator $\psi(y)$ $x, y \in \Lambda \subset \mathbb{R}^{3}$, which satisfy the commutation relationships of the bose- and fermi-type:

$$
\begin{aligned}
& {[\psi(x), \psi(y)]_{\mp}=\left[\psi^{+}(x), \psi^{+}(y)\right]_{\mp}=0,} \\
& {\left[\psi(x), \psi^{+}(y)\right]_{\mp}=\delta(x-y)}
\end{aligned}
$$

As a basic algebra [7,13,14,26-29] of the observed quantities, which describes the interaction of the particles in system, so called the current Lie-algebra $\mathcal{G}$ is chosen. To construct it let us introduce the following basic operator quantities:

$$
\rho(x)=\psi^{+}(x) \psi(x)
$$


is the operator of the density of the particles;

$$
j(x)=\frac{1}{2 i}\left[\psi^{+}(x), \nabla_{x} \psi(x), \nabla_{x} \psi^{+}(x) \psi(x)\right]
$$

is the operator of the particle flow density in the point $x \in \Lambda$. It is easy to check that operators

$$
\rho(f)=\int_{\Lambda} d x f(x) \rho(x), \quad j(g)=\int_{\Lambda} d x\langle g(x) \mid j(x)\rangle,
$$

where $f \in \mathcal{J}(\Lambda ; \mathbb{R}), g \in \mathcal{J}\left(\Lambda ; \mathbb{R}^{3}\right)$, are rapidly decreasing functions of the Schwartz-type, $\langle\cdot \mid \cdot\rangle$ is a scalar product in $\mathbb{R}^{3}$, which satisfy the following commutation relationships:

$$
\begin{aligned}
& {\left[\rho\left(\mathrm{f}_{1}\right), \rho\left(\mathrm{f}_{2}\right)\right]=0, \quad[\rho(\mathrm{f}), j(\mathrm{~g})]=i \rho(\mathrm{g}, \nabla \mathrm{f}),} \\
& {\left[j\left(\mathrm{~g}_{1}\right), j\left(\mathrm{~g}_{2}\right)\right]=i j\left(\left[\mathrm{~g}_{1}, \mathrm{~g}_{2}\right]\right), \quad\left[\mathrm{g}_{1}, \mathrm{~g}_{2}\right]=\mathrm{g}_{1}^{\prime} \mathrm{g}_{2}-\mathrm{g}_{2}^{\prime} \mathrm{g}_{1}}
\end{aligned}
$$

for all $\mathrm{f}, \mathrm{f}_{1}, \mathrm{f}_{2} \in \mathcal{J}(\Lambda ; \mathbb{R}), \mathrm{g}, \mathrm{g}_{1}, \mathrm{~g}_{2} \in \mathcal{J}\left(\Lambda ; \mathbb{R}^{3}\right)$. Current Lie algebra $\mathcal{G}$ (22) is a basic object in the algebraic approach to the theory of the Bogolubov's quantum generating functional. By the general physics principle any observable quantity belongs to the generalized universal enveloping algebra $\mathcal{A}(\mathcal{G})$, generated [30] by the basis current algebra $\mathcal{G}$ (22). It is obvious, that operators (21) are self-adjoint in $\Phi$, that is $\rho^{+}(f)=j(f), j^{+}(g)=j(g), f \in \mathcal{J}(\Lambda ; \mathbb{R})$, $g \in \mathcal{J}\left(\Lambda ; \mathbb{R}^{3}\right)$, but in general case they can be unbounded, that is why it is natural to deal with the elements $U(f)$ and $W\left(\phi_{t}^{g}\right)$, which are defined as $U(f)=\exp \{i \rho(f)\}$, $W\left(\phi_{t}^{g}\right)=\exp \{i t j(g)\}$ and generate group with the following composition rules:

$$
\begin{aligned}
& U\left(f_{1}\right) \cdot U\left(f_{2}\right)=U\left(f_{1}+f_{2}\right), \\
& W\left(\phi_{1}\right) \cdot W\left(\phi_{2}\right)=W\left(\phi_{1}+\phi_{2}\right), \\
& W(\phi) \cdot U(f)=U(f \cdot \phi) W(\phi)
\end{aligned}
$$

for all $f \in \mathcal{J}(\Lambda ; \mathbb{R}), g \in \mathcal{J}\left(\Lambda ; \mathbb{R}^{3}\right)$, and $\frac{d}{d t} \phi_{t}^{g}=g \cdot \phi_{t}^{g}(x), \phi_{0}^{g}=x \in \Lambda, g \cdot \phi_{t}^{g}(x)=$ $g\left(\phi_{t}^{g}(x)\right)$, where $t \in \mathbb{R}$ is a parameter. Group $G(23)$ is a semi-direct product $\operatorname{Diff}(\Lambda) \ltimes$ $\mathcal{J}(\Lambda ; \mathbb{R})$, where $\operatorname{Diff}(\Lambda)$ is a diffeomorphisms group of the domain $\Lambda \subset \mathbb{R}^{3}$. The group rule, according to (23), has the following form:

$$
\left(\phi_{1}, f_{1}\right) \cdot\left(\phi_{2}, f_{2}\right)=\left(\phi_{2} \cdot \phi_{1}, f_{1}+f_{2} \cdot \phi_{1}\right),
$$

where $(\phi, f) \in G=\operatorname{Diff}(\Lambda) \ltimes \mathcal{J}(\Lambda)$ is an arbitrary element of the current group. The diffeomorphisms group $\operatorname{Diff}(\Lambda)$ in $G$ as a topological space is an addition of the metrized locally linear connected (but not locally compact) subgroup of the smooth diffeomorphisms $\operatorname{Diff}_{0}(\Lambda)$ of the domain $\Lambda \subset \mathbb{R}^{3}$ with the compact supports and standard group operation with composition of mappings.

It is well known [7] that different unitary representations of the current algebra $G=\operatorname{Diff}(\Lambda) \ltimes \mathcal{J}(\Lambda ; \mathbb{R})$ describe different many-particle physical systems. For example, system of the $N$ identical Fermi-particles and system of identical Bose-particles correspond to two unitary nonequivalent representations of this group. Since the group $G$ is infinitely parametric, than its different possible unitary representations describe wide spectrum of the physics problems $[7,30]$.

The Hilbert space $\Phi$ for every irreducible unitary representation of the current group $G=\operatorname{Diff}(\Lambda) \ltimes \mathcal{J}(\Lambda ; \mathbb{R})$ is unitary equivalent [7] to the Hilbert space

$$
\Phi_{\mu}=\oplus \int_{\mathcal{J}^{\prime}} d \mu(F) \Phi_{F}
$$

where $\mu$ is a cylindrical measure on the space $\mathcal{J}^{\prime}$ of the continuous real linear functionals (distributions) on $\mathcal{J}$ [31,32], $\phi_{F}$ are marked by the index $F \in \mathcal{J}^{\prime}$ complex linear spaces. 
In physics application it is frequently put $\operatorname{dim} \Phi_{F}=1$, then $\Phi_{\mu} \simeq L_{2}^{(\mu)}$ is a space of quadratically integrable functions on $\mathcal{J}^{\prime}$ with measure $\mu$.

Let us now take the arbitrary element $\omega(F) \in \Phi_{\mu}$. Then the group $G=\operatorname{Diff}(\Lambda) \ltimes \mathcal{J}$ action on this element has the following representation:

$$
\begin{aligned}
U(f) \omega(F) & =\exp (i F(f)) \omega(F), \\
W(\phi) \omega(F) & =\chi_{\phi}(F) \omega\left(\phi^{*} F\right)\left[\frac{d \mu\left(\phi^{*} F\right)}{d \mu(F)}\right]^{1 / 2},
\end{aligned}
$$

where $\phi^{*} F(\mathrm{f})=F(\mathrm{f} \cdot \phi), \mathrm{f} \in \mathcal{J}, \phi \in \operatorname{Di} f f(\Lambda), F \in \mathcal{J}^{\prime}$,

$$
\chi_{\phi_{1}}(F) \chi_{\phi_{2}}\left(\phi_{1}^{*} F\right)=\chi_{\phi_{1} \cdot \phi_{2}}(F)
$$

for all $\phi_{1}, \phi_{2} \in \operatorname{Dif} f(\Lambda)$. For the Radon-Nikodym derivative to exist in (26) the measure $\mu$ on $\mathcal{J}^{\prime}$ should be quasi-invariant $[13,14,31,33]$ with respect to the group $\operatorname{Di} f f(\Lambda)$, that is for any measurable set $Q \in \mathcal{J}^{\prime}$ and arbitrary $\phi \in \operatorname{Dif} f(\Lambda)$ measure $\mu(Q)=0$ iff $\mu\left(\phi^{*} Q\right)=0$.

The representation (26), which corresponds to the quantum-chemical system $N \in \mathbb{Z}$ identical particles, possesses measure $\mu$, supported on the Dirac delta-function, i.e., on the functionals

$$
F=\sum_{j=1}^{N} \delta\left(x-x_{j}\right)
$$

with the measure

$$
d \mu(F)=\Omega^{*} \Omega \prod_{j=1}^{N} d x_{j} \delta\left(F-\sum_{j=1}^{N} \delta\left(x-x_{j}\right)\right),
$$

where $x, x_{j} \in \Lambda, j=\overline{1, N}$, and $\Omega \in L_{2}^{ \pm}\left(\Lambda^{N} ; \mathbb{C}\right)$ is a symmetrical or anti-symmetrical wave function of the base state of the $N$-particle dynamical systems. The following equalities hold:

$$
\Omega(F)=1, \quad \omega \in L_{\Lambda}^{(\mu)}\left(\mathcal{J}^{\prime} ; \mathbb{C}\right)
$$

and

$$
\begin{aligned}
& \rho(x) \omega(F)=\sum_{j=1}^{N} \delta\left(x-x_{j}\right) \omega(F), \\
& j(x) \omega(F)=\frac{1}{2 i} \sum_{j=1}^{N}\left[\delta\left(x-x_{j}\right) \cdot \nabla_{j}+\nabla_{j} \cdot \delta\left(x-x_{j}\right)\right],
\end{aligned}
$$

where $\omega(F)=\omega\left(x_{1}, x_{2}, \ldots, x_{N}\right) \in \Phi_{\mu} \simeq L_{2}^{ \pm}\left(\Lambda^{N} ; \mathbb{C}\right), x, x_{j} \in \Lambda, j=\overline{1, N}$. From (30) and (26) we can find the following relationships:

$$
\begin{aligned}
& U(f) \omega(F)=\exp \left[i \sum_{j=1}^{N} f(x, j)\right] \omega(F) \\
& W(f) \omega(F)=\omega\left(\phi^{*} F\right)\left[\operatorname{det}\left|\frac{\partial \Phi(x)}{\partial x}\right|\right]^{1 / 2},
\end{aligned}
$$

where we put $\chi_{\phi}(F)=1$ for all $\phi \in \operatorname{Diff}\left(\mathbb{R}^{3}\right), \omega\left(\phi^{*} F\right)=\omega\left(\phi x_{1}, \ldots, \phi x_{N}\right)$ in the case of Bose statistics, $x_{j} \in \Lambda, j=\overline{1, N}$.

Let us consider the representation $\pi: G \rightarrow A u t\left(\Phi_{\mu}\right)$ of the current group $G=$ $\operatorname{Diff}(\Lambda) \ltimes \mathcal{J}(\Lambda ; \mathbb{R})$, limited on the Abel subgroup $\mathcal{J}$. Then the corresponding Bogolubovtype generation functional [7] can be defined in the form

$$
\mathcal{L}(f)=(\Omega \mid \exp [i \rho(f)] \Omega)=\int_{\mathcal{J}^{\prime}} d \mu(F) \exp [i F(f)],
$$


where cyclic vector $\Omega \in \Phi_{\mu}$ of the representation is normed in $\Phi_{\mu}$ as $(\Omega \mid \Omega)=1$. The generation functional (32) has wide use in the case of the representation of the current Lie algebra $\mathcal{G}$ (22), which corresponds to the stationary (equilibrium) statistical state of the original many-particle dynamical system [7] and satisfy the following functional Bogolubov-type equation [34]:

$$
\left[\nabla_{x}-i \nabla f(x)\right] \frac{1}{2 i} \frac{\delta \mathcal{L}(f)}{\delta f(x)}=A\left(x ; \frac{1}{i} \frac{\delta}{\delta f(x)}\right) \mathcal{L}(f)
$$

Here, $A\left(x ; \frac{1}{i} \frac{\delta}{\delta f(x)}\right)$ is the so-called characteristic operator of the representation $\pi$ : $G \rightarrow \operatorname{Aut}\left(\Phi_{\mu}\right)$, defined by the following operator relationship:

$$
K(x) \Omega=A(x ; \rho) \Omega,
$$

where $x \in \Lambda$ and

$$
K(x)=\nabla_{x} \rho(x) / 2+i j(x)=\psi^{+}(x) \nabla_{x} \psi(x)
$$

are the elements of the enveloping algebra $A(\mathcal{G})$. In the case of equilibrium statistical system state at inverse temperature $\beta \rightarrow 0$ the operator $A(x ; \rho)$ was first built by N. Bogolubov [34] by means of indirect method:

$$
A(x ; \rho)=-\frac{\beta}{2} \int_{\Lambda^{N}} d y \nabla_{x} V(x-y): \rho(x) \rho(y):,
$$

where : : is a standard normal operator ordering [3]:

$$
: \rho\left(x_{1}\right) \ldots \rho\left(x_{n}\right):=\prod_{j=1}^{N}\left(\rho\left(x_{j}\right)-\sum_{k=1}^{j-1} \delta\left(x_{j}-x\right),\right)
$$

where $x_{j} \in \Lambda, j=1, \ldots, n, n \in \mathbb{Z}_{+}$, is arbitrary and $V(x-y)$ for $x, y \in \Lambda$ is a potential of two-particle interaction in the dynamical system. Taking into account, that

$$
f_{n}\left(x_{1}, \ldots, x_{n}\right)=: \frac{1}{i} \frac{\delta}{\delta f\left(x_{1}\right)} \ldots \frac{1}{i} \frac{\delta}{\delta f\left(x_{n}\right)}:\left.\mathcal{L}(f)\right|_{f=0}
$$

are Bogolubov's $n$-particle distribution functions [34], $n \in \mathbb{Z}_{+}$, from (33) it is easy to obtain unlimited hierarchy of the Bogolubov's equations for the function (38), whose solutions are important for the physics applications.

As it was found in [34], the functional Equation (33) does not have a unique solution, that is why there posed the problem of choosing the solution with physical sense. This problem is solved in [7] by means of the functional representation operators of the Bloch equation in form of the additional compatible with (33) functional equation on Bogolubov's functional $\mathcal{L}(f), f \in \mathcal{J}$.

In the case of non-stationary (non-equilibrium) states of the many-particle dynamical systems the Bogolubov's generating functional (32) doesn't possess all needed information. We introduce the following Bogolubov's type generating functional:

$$
\mathcal{L}(f, g)=(\Omega \mid \exp [i \rho(f)] \exp [i f(g)] \Omega)=\operatorname{Tr}(P \exp [i \rho(f)] \exp [i j(g)]) .
$$

Here, $\Omega \in \Phi_{\mu}$ is a cyclic vector of the representation of the current group $G$, which satisfies the following conditions:

$$
T \rho(f) T^{-1}=\rho(f), \quad T \Omega=\Omega^{*}, \quad T j(g) T^{-1}=-j(g), \quad T \mathbb{H} T^{-1}=\mathbb{H},
$$

where operator $T: \mathbb{R} \ni t \rightarrow-t \in \mathbb{R}$ is an operator of the time inversion, $f \in \mathcal{J}(\Lambda ; \mathbb{R})$ and $g \in \mathcal{J}\left(\Lambda ; \mathbb{R}^{3}\right)$ are arbitrary. 
In $N$-particle representation of the Lie-algebra $\mathcal{G}$ (30), $N \in \mathbb{Z}_{+}$, the functional $\mathcal{L}(f, g)(39)$ has the following form:

$$
\begin{aligned}
\mathcal{L}(f, g) & =\int_{\Lambda} d x_{1} \ldots \int_{\Lambda} d x_{N} \Omega^{*}\left(x_{1}, \ldots, x_{N}\right) \prod_{j=1}^{N} \exp \left[i f\left(x_{j}\right)\right] \times \\
& \times \exp \left[i \xi\left(x_{j}, g\right)\right] \Omega\left(x_{1}, \ldots, x_{N}\right)
\end{aligned}
$$

where $\xi(x, g)=\frac{1}{2 i}\left[g(x) \nabla_{x}+\nabla_{x} g(x)\right], x \in \Lambda$, and $\Omega \in L_{2}^{ \pm}\left(\Lambda^{N} ; \mathbb{C}\right)$ is a cyclic state. The operator $\exp [i \xi(x, g)]$ acts on any function $\omega \in L_{2}^{ \pm}\left(\Lambda^{N} ; \mathbb{C}\right)$ by the rule:

$$
\exp [i \xi(x, g)] \omega\left(x_{1}, \ldots, x_{N}\right)=\left(\phi^{*} \omega\right)\left(x_{1}, \ldots, x_{N}\right)\left[\operatorname{det}\left\|\frac{\partial \phi(x)}{\partial x}\right\|\right]^{1 / 2}
$$

where $\phi \in \operatorname{Diff}(\Lambda)$ is a diffeomorphism $\Lambda$, corresponding to the vector field $g \in \mathcal{J}\left(\Lambda ; \mathbb{R}^{3}\right)$, that is $\phi(x)=\phi_{t}^{\mathcal{G}}$, where $\frac{d}{d t} \phi_{t}^{\mathcal{G}}=g\left(\phi_{t}^{\mathcal{G}}(x)\right), x \in \Lambda$. For $N \rightarrow \infty$ the expression (40) becomes

$$
\begin{aligned}
& \mathcal{L}(f, g)=\sum_{n \in \mathbb{Z}_{+}} \frac{1}{n !} \int_{\Lambda} d x_{1} \ldots \int_{\Lambda} d x_{n} \int_{\Lambda} d y_{1} \ldots \int_{\Lambda} d y_{n} \prod_{j=1}^{n}\left[\delta\left(x_{j}-y_{j}\right) \times\right. \\
& \left.\times\left\{\exp \left[i f\left(x_{j}\right)\right] \exp \left[i \xi\left(x_{j}, g\right)\right]-1\right\} f_{n}\left(y_{1}, \ldots, y_{n} ; x_{1}, \ldots, x_{n}\right)\right]
\end{aligned}
$$

where for all $n \in \mathbb{Z}_{+}$Bogolubov's quantum distribution functions [3] are

$$
f_{n}\left(y_{1}, \ldots, y_{n} ; x_{1}, \ldots, x_{n}\right)=\left(\Omega, \psi^{+}\left(y_{n}\right) \ldots \psi^{+}\left(y_{1}\right) \psi\left(x_{1}\right) \ldots \psi\left(x_{n}\right) \Omega\right) .
$$

It is obvious that

$$
: f_{n}\left(x_{1}, \ldots, x_{n}\right):=f_{n}\left(x_{1}, \ldots, x_{n} ; x_{1}, \ldots, x_{n}\right),
$$

where $x_{j} \in \Lambda, j=\overline{1, n}, n \in \mathbb{Z}_{+}$.

To proceed with the further study of the classical distribution functions of the manyparticle dynamical system (that is, $\beta \rightarrow 0$, and the Planck constant $\hbar \rightarrow 0$ ), let us introduce the following quantized Wigner operator $w(x, p): \Phi_{\mu} \rightarrow \Phi_{\mu}$, according to the works in $[1,5,11,35]$ :

$$
w(x, p)=\frac{1}{(2 \pi)^{n}} \int_{\Lambda} d \alpha \exp (i\langle\alpha \mid p\rangle) \psi^{+}\left(x+\frac{\hbar \alpha}{2}\right) \psi\left(x-\frac{\hbar \alpha}{2}\right),
$$

where $(x, p) \in T^{*}(\Lambda)$. Performing transformation (44) in the expression (39), we can find, that

$$
\begin{gathered}
\mathcal{L}(f, g) \rightarrow \mathcal{L}(f)=\sum_{n \in \mathbb{Z}_{+}} \frac{1}{n !} \int_{T^{*}(\Lambda)} d x_{1} d p_{1} \int_{T^{*}(\Lambda)} d x_{2} d p_{2} \ldots \times \\
\times \int_{T^{*}(\Lambda)} d x_{n} d p_{n} \prod_{j=1}^{n}\left\{\exp \left(i f\left(x_{j}, p_{j}\right)\right)-1\right\} f_{n}\left(x_{1}, p_{1} ; \ldots ; x_{n}, p_{n}\right),
\end{gathered}
$$

where $f \in \mathcal{J}\left(T^{*}(\Lambda) ; \mathbb{R}\right)$. From the expression (45) it also follows that

$$
\mathcal{L}(\mathrm{f})=(\Omega \mid \exp [i w(\mathrm{f})] \Omega)=\operatorname{Tr}(P \exp [i w(\mathrm{f})])
$$

where $w(\mathrm{f})=\int_{T^{*}(\Lambda)} d x d p w(x, p) \mathrm{f}(x, p), P: \Phi_{\mu} \rightarrow \Phi_{\mu}$ is the corresponding Gibbs statistical operator. The corresponding quantum current Lie algebra transforms into the abelian Lie algebra of the operator functionals $\left\{w(\mathrm{f}) \in \mathcal{G}: \mathrm{f} \in \mathcal{J}\left(T^{*}(\Lambda) ; \mathbb{R}\right)\right\}$ in a weak functional sense. This property will be used in the next sections. 


\subsection{The Bogolubov's Functional Equations in Non-Equilibrium Statistical Mechanics}

Let us be given the quantum dynamical system of the many identical particles with the average density $\bar{\rho}=\lim _{\Lambda \nearrow \mathbb{R}^{3}}(N / A) \in \mathbb{R}_{+}^{1} \backslash\{0\}, \lim N \rightarrow \infty$ and $\Lambda \nearrow \mathbb{R}^{3}$ in Van Hove's sense $[5,6]$. Then, according to $[7,13,14]$, the Hamiltonian operator in the second quantization representation [3] is given by the expression

$$
\begin{aligned}
\mathbb{H} & =\frac{\hbar^{2}}{2 m} \int_{\Lambda} d x\left\langle\nabla \psi^{+}(x) \mid \nabla \psi(x)\right\rangle+\frac{1}{2} \int_{\Lambda} d x \int_{\Lambda} d y \mathrm{~V}(x-y) \times \\
& \times \psi^{+}(x) \psi^{+}(y) \psi(y) \psi(x),
\end{aligned}
$$

where $\Phi(x-y)$ is translationary invariant potential of the interparticle interaction, $m \in$ $\mathbb{R}_{+}^{1} \backslash\{0\}$ is a mass of one particle. In terms of operators of the current Lie algebra (22), the operator $(47)$ can be rewritten $[7,13,14]$ as

$$
\mathbb{H}=\frac{\hbar^{2}}{2 m} \int_{\Lambda} d x\left\langle K^{+}(x) \mid \rho^{-1}(x) K(x)\right\rangle+\frac{1}{2} \int_{\Lambda} d x \int_{\Lambda} d y \mathrm{~V}(x-y): \rho(x) \rho(y):,
$$

where the operator $K(x): \Phi_{\mu} \rightarrow \Phi_{\mu}$ is given by the expression (44). Then, we can find the expression for the Wigner representation (44) for the Hamiltonian operator (47):

$$
\mathbb{H}=\int_{T^{*}(\Lambda)} d z \frac{p^{2}}{2 m} w(z)+\int_{T^{*}(\Lambda)} d z \int_{T^{*}(\Lambda)} d z^{\prime} \mathrm{V}\left(x-y^{\prime}\right): w(z) w\left(z^{\prime}\right):
$$

where $z=(x, p) \in T^{*}(\Lambda), z^{\prime}=(y, q) \in T^{*}\left(\Lambda\right.$ and $d z=d x d p, d z^{\prime}=d y d q$ are the standard volume measures in $T^{*}(\Lambda)$. According to the Heisenberg's principle [3], the evolution equation by the variable $t \in \mathbb{R}_{+}$for the arbitrary operator for the observable quantity $a: \Phi \rightarrow \Phi$ is

$$
\frac{d a}{d t}=\frac{i}{\hbar}[\mathbb{H}, a],
$$

where $[\cdot, \cdot]$ is a usual operator commutator. Following [3], it is easy to state, that for $\hbar \rightarrow 0$ in the weak sense the following theorem is true.

Theorem 1. Let us $\mathcal{M}$ is an algebra of the self-adjoint operators with $A(\mathcal{G})$ in the Wigner representation. Then operator bracket $[\cdot, \cdot]_{0}=\lim _{\hbar \rightarrow 0}[\cdot, \cdot]$ on the algebra $\mathcal{M}$ in the weak sense is equivalent to

$$
\begin{aligned}
{\left[a_{j}, a_{n}\right]_{0} } & =\sum_{k=1}^{\min \{j, n\}} \int_{T^{*}(\Lambda)} d z_{1} \ldots \int_{T^{*}(\Lambda)} d z_{k}: w\left(z_{1}\right) \ldots w\left(z_{k}\right) \times \\
& \times\left\{\frac{\delta^{k} a_{j}}{\delta w\left(z_{1}\right) \ldots \delta w\left(z_{k}\right)}, \frac{\delta^{k} a_{n}}{\delta w\left(z_{1}\right) \ldots \delta w\left(z_{k}\right)}\right\}^{(k)},
\end{aligned}
$$

where $\{\cdot, \cdot\}^{(k)}$ is a standard canonical Poisson bracket on the phase space of $k \in \mathbb{Z}_{+}$particles.

The statement (51) could be proved by means of the next general Bohr-Dirac correspondence principle in the quasi-classical approach:

$$
\lim _{\hbar \rightarrow 0} \frac{i}{\hbar}[a, b]=\{a, b\}^{(N)},
$$

where $N$ is a maximal number of the particle in system and $a, b \in A(\mathcal{G})$ are operators in $N$-particle Hilbert space representation $\Phi_{\mu}=L_{2}^{ \pm}\left(\Lambda^{N} ; \mathbb{C}\right), F=\sum_{j=1}^{N} \delta\left(x-x_{j}\right)$. 
Corollary 1. Algebra of the operators of the observable quantities $A(\mathcal{G})$ for $\hbar \rightarrow 0$ allows "hierarchical" representation

$$
A(\mathcal{G})=\sum_{j \in \mathbb{Z}_{+}} A_{j}(\mathcal{G}) \Rightarrow \mathcal{M}=\underset{j=\mathbb{Z}_{+}}{\oplus} A_{j}(\mathcal{G})
$$

along with Lie bracket $\llbracket \cdot, \cdot \rrbracket$, which is inducted by the bracket $[\cdot, \cdot]_{0}$ (51):

$$
\llbracket a, b \rrbracket=\bigoplus_{l \in \mathbb{Z}_{+}} \sum_{j, k \in \mathbb{Z}_{+}}\left[a_{j}, b_{k}\right]_{0}^{(l)}
$$

where $a, b \in \mathcal{M}$ in the Wigner representation and the following expansions hold

$$
a=\sum_{j \in \mathbb{Z}_{+}} a_{j}, \quad b=\sum_{j \in \mathbb{Z}_{+}} b_{j}, \quad\left[a_{j}, b_{k}\right]_{0}=\sum_{l \in \mathbb{Z}_{+}}\left[a_{j}, b_{k}\right]_{0}^{(l)}
$$

Let us consider the following linear mapping $\alpha: \mathcal{M} \rightarrow A(\mathcal{G})$, where

$$
\alpha\left(\underset{j \in \mathbb{Z}_{+}}{\oplus} a_{j}\right)=\sum_{j=\mathbb{Z}_{+}} a_{j} \in A(\mathcal{G}),
$$

and the Lie bracket $\llbracket \cdot, \cdot \rrbracket$ is defined in $\mathcal{M}$, and the corresponding Lie bracket $[\cdot, \cdot]_{\alpha}(51)$ in $A(\mathcal{G})$. Let us consider the dual to (56) mapping $\alpha^{*}: A(\mathcal{G})^{*} \rightarrow \mathcal{M}^{*}$, where

$$
\begin{aligned}
\mathcal{M}^{*} & =\underset{l \in \mathbb{Z}_{+}}{\oplus} \mathcal{M}_{j}^{*}, \quad \mathcal{M}=\underset{l \in \mathbb{Z}_{+}}{\oplus} \mathcal{M}_{j}, \\
\mathcal{M}^{*} & =\sum_{j \in \mathbb{Z}_{+}}\left\{P \in A_{j}(\mathcal{G})^{*}: F(a)=\operatorname{Tr}(P a), a \in A(\mathcal{G})\right\} .
\end{aligned}
$$

Here, $P: \Phi_{\mu} \rightarrow \Phi_{\mu}$ is statistic operator of the initial dynamical system (49), which satisfy the Heisenberg-Liouville equation

$$
\frac{d P}{d t}=\frac{i}{\hbar}[P, \mathbb{H}]
$$

for all $t \in \mathbb{R}_{+}$. When $\hbar \rightarrow 0$ the expression (58), according to (52), transforms into quasi-classical Liouville equation in the Wigner representation.

It is easy to check, that for element $F \in A(\mathcal{M})^{*}$ the expression

$$
\alpha^{*} F=\left(f_{1}, \ldots, f_{j}, \ldots\right)=\mathcal{F} \in \mathcal{M}^{*}
$$

defines the representation on the space $\mathcal{M}^{*}$ of the distribution functions

$$
f_{j}=\operatorname{Tr}\left(P: w\left(x_{1}\right) \ldots w\left(z_{j}\right):\right),
$$

where $j \in \mathbb{Z}_{+}$and for any $a \in \mathcal{M}$

$$
a \mathcal{F}=\sum_{j \in \mathbb{Z}_{+}} \int_{T^{*}(\Lambda)} d z_{1} \ldots \int_{T^{*}(\Lambda)} d z_{j} f_{j}\left(z_{1}, \ldots, z_{j}\right) a_{j}\left(z_{1}, \ldots, z_{j}\right) .
$$

Let $b(F), c(F) \in D\left(A(\mathcal{G})^{*}\right)$ be linear functionals on $A(\mathcal{G})^{*}$, then on $D\left(A(\mathcal{G})^{*}\right)$ there is defined standard [7] Lie-Poisson bracket $\{\cdot, \cdot\}_{0}$ via the rule

$$
\{b(F), c(F)\}_{0}=F\left([b, c]_{0}\right),
$$


where $b, c \in A(\mathcal{G})$ are such that $F(b)=b(F), F(c)=c(F), F \in A^{*}(\mathcal{G})$. In the same way the dual Lie-Poisson bracket $\{\{\cdot, \cdot\}\}$ is defined on the set of functionals $D^{*}(\mathcal{M})$ over the adjoint space $\mathcal{M}^{*}(57)$

$$
\{\{b(\mathcal{F}), c(\mathcal{F})\}\}=F(\llbracket b, c \rrbracket),
$$

where $F(b)=b(F), F(c)=c(F), F \in \mathcal{M}^{*}$.

Definition 1. It is said, that mapping of the Lie algebras $\alpha: \mathcal{M} \rightarrow A(\mathcal{G})$ is canonical (or Poissonian [7]), if for all $b(\mathcal{F})$ and $c(\mathcal{F})$ the following equality holds:

$$
\alpha^{*}\{b(F), c(F)\}_{0}=\left\{\left\{\alpha^{*} b(F), \alpha^{*} c(F)\right\}\right\},
$$

where $\mathcal{F}=\alpha^{*} F \in \mathcal{M}^{*}$

From the reasoning above, we can formulate the following proposition.

Proposition 1. Let $A$ and $\mathcal{M}$ are two arbitrary Lie algebras, and $\alpha: \mathcal{M} \rightarrow A$ is a linear mapping. Then, dual mapping $\alpha^{*}: D\left(A(\mathcal{G})^{*}\right) \rightarrow D\left(\mathcal{M}^{*}\right)$ is canonical iff $\alpha: \mathcal{M} \rightarrow A$ is Lie algebras homomorphism.

As a consequence of the statement above, one derives the next theorem.

Theorem 2. Dual mapping $\alpha^{*}: D\left(A(\mathcal{G})^{*}\right) \rightarrow D\left(\mathcal{M}^{*}\right)$, which was built by means of the hierarchical Lie algebra of the operators $\mathcal{M}$, is canonical.

Let us consider the generating functional $\mathcal{L}(f), f \in \mathcal{J}\left(T^{*}(\Lambda) ; \mathbb{R}\right),(46)$ in Wigner representation and apply the developed above algebraic technique to the calculation of the following quantity:

$$
\frac{d}{d t} \mathcal{L}(f)=\frac{i}{\hbar} \operatorname{Tr}(P(\mathbb{H} \exp [i w(f)]))
$$

when $\hbar \rightarrow 0$ and $t \in \mathbb{R}$.

From (38) and (45), one can find that

$$
\frac{d}{d t} \mathcal{L}(f)(\mathcal{F})=\operatorname{Tr}\left(P\left[\mathbb{H}: \exp \left[i w\left(e^{i f}-1\right)\right]:\right]\right)=\alpha^{*}\{\mathcal{H}(\mathcal{F}), \mathcal{L}(f)(\mathcal{F})\}_{\alpha},
$$

where for all $F \in A(\mathcal{G})^{*}$ the Hamiltonian functional $\mathcal{H}(\mathcal{F}) \in D\left(A(\mathcal{G})^{*}\right)$ is given as

$$
\begin{aligned}
\mathcal{H}(\mathcal{F}) & =\operatorname{Tr}\left(P(\mathbb{H}(F))=\int_{T^{*}(\Lambda)} d z T(p) f_{1}(z)+\right. \\
& +\frac{1}{2} \int_{T^{*}(\Lambda)} d z_{1} \int_{T^{*}(\Lambda)} d z_{2} \mathrm{~V}\left(x_{1}-x_{2}\right) f_{2}\left(z_{1}, z_{2}\right) .
\end{aligned}
$$

From (66) and Theorem 2, we immediately obtain that

$$
\frac{d}{d t} \mathcal{L}(f)(\mathcal{F})=\{\{\mathcal{L}(f)(\mathcal{F}), \mathcal{H}(\mathcal{F})\}\},
$$

where $t \in \mathbb{R}, \mathcal{L}(f)(\mathcal{F})=\alpha^{*} \mathcal{L}(f)(F), \mathcal{H}(\mathcal{F})=\alpha^{*} \mathbb{H}(F)$ and $\mathcal{F} \in \mathcal{M}^{*}$ is arbitrary.

Thus, the following theorem is stated.

Theorem 3. The generating functional of the Bogolubov's distribution functions $\mathcal{L}(f)(\mathcal{F})(46)$ on the phase space $D(\mathcal{M})$ satisfies the Hamiltonian dynamical system (68) with the Lie-Poisson bracket (63) and Hamiltonian function (67) taken as functional on $\mathcal{M}^{*}$. 
Using Equation (68) and formulae (51) and (54), we finally get the following nonequilibrium functional Bogolubov's equation [2]:

$$
\begin{aligned}
& \frac{d}{d t} \mathcal{L}(f)=\int_{T^{*}(\Lambda)} d z\left\{\frac{1}{i} \frac{\delta \mathcal{L}(f)}{\delta f(z)}, T(p)\right\}^{(1)}+ \\
& +\frac{1}{2} \int_{T^{*}(\Lambda)} d z_{1} \int_{T^{*}(\Lambda)} d z_{2}\left\{: \frac{1}{i} \frac{\delta}{\delta f\left(z_{1}\right)} \frac{1}{i} \frac{\delta}{\delta f\left(z_{2}\right)}:, \mathrm{V}\left(x_{1}-x_{2}\right)\right\}^{(2)} \mathcal{L}(f),
\end{aligned}
$$

where for any $n \in \mathbb{Z}_{+}$according to (37)

$$
: \frac{1}{i} \frac{\delta}{\delta f\left(z_{1}\right)} \cdots \frac{1}{i} \frac{\delta}{\delta f\left(z_{n}\right)}:=\prod_{j=1}^{n}\left[\frac{1}{i} \frac{\delta}{\delta f\left(z_{j}\right)}-\sum_{k=1}^{j} \delta\left(z_{j}-z_{k}\right)\right]
$$

and, by definition, $\{\cdot, \cdot\}^{(j)}$ denotes the standard canonical Poisson bracket on the phase space $T^{*}(\Lambda)^{j}$ for all $j \in \mathbb{Z}_{+}$.

Taking into account, that for functional $\mathcal{L}(f), f \in \mathcal{J}\left(T^{*}(\Lambda) ; \mathbb{R}\right)$, there exists the unlimited expansion (45):

$$
\mathcal{L}(f)=\sum_{n \in \mathbb{Z}} \frac{1}{n !} \int_{T^{*}(\Lambda)} d z_{1} \ldots \int_{T^{*}(\Lambda)} d z_{n} \prod_{i=1}^{n}\left\{\exp \left[i f\left(z_{j}\right)-1\right]\right\} f_{n}\left(z_{1}, \ldots, z_{n}\right),
$$

from (69) we obtain the kinetic equations for the hierarchy of the Bogolubov's distribution functions [2]:

$$
\begin{aligned}
& \frac{\partial}{\partial t} f_{n}\left(z_{1}, \ldots, z_{n}\right)=\left\{f_{n}\left(z_{1}, \ldots, z_{n}\right), H_{n}\left(z_{1}, \ldots, z_{n}\right)\right\}^{(n)}+ \\
& +\int_{T^{*}(\Lambda)} d z_{1} \ldots \int_{T^{*}(\Lambda)} d z_{n+1}\left\{f_{n}\left(z_{1}, \ldots, z_{n+1}\right), H_{n}\left(z_{1}, \ldots, z_{n+1}\right), \sum_{j=1}^{n} \mathrm{~V}\left(x_{j}-x_{n+1}\right)\right\}^{(n+1)},
\end{aligned}
$$

where $z_{j} \in \Lambda, j=1, \ldots, n$, are the coefficients of the $n$-particle cluster in $\Lambda, H_{n}\left(z_{1}, \ldots, z_{n}\right)$ is its corresponding energy:

$$
H_{n}\left(z_{1}, \ldots, z_{n}\right)=\sum_{j=1}^{n} \frac{p_{j}^{2}}{2 m}+\frac{1}{2} \sum_{j \neq k=1}^{n} \mathrm{~V}\left(x_{j}-x_{k}\right)
$$

Thus, the problem of the construction of the kinetic theory by Bogolubov reduced to the finding the special solutions of the unlimited hierarchy of the Equations (72), where the selection criterion is based on the Bogolubov's fundamental weakening correlation principle:

$$
\lim _{\|\langle n\rangle-\langle m\rangle\| \rightarrow \infty}\left|f_{n+m}\left(z_{1}, \ldots, z_{n+m}\right)-f_{n}\left(z_{1}, \ldots, z_{n}\right) f_{m}\left(z_{n+1}, \ldots, z_{n+m}\right)\right| \rightarrow 0
$$

where $\|\langle n\rangle-\langle m\rangle\|=\operatorname{dist}\left(\left\{z_{i} \in T^{*}(\Lambda): i=1, \ldots, n\right\},\left\{z_{i+n} \in T^{*}(\Lambda): i=1, \ldots, m\right\}\right)$ is a distance between two clusters with $n \in \mathbb{Z}_{+}$and $m \in \mathbb{Z}_{+}$numbers of the particles.

If there exists a special solution of the hierarchy (67) in the functional form

$$
f_{n}\left(z, \ldots, z_{n} ; t\right)=f_{n}\left(z_{1}, \ldots, z_{n} ; f_{1}(z ; t)\right)
$$

for all $t \in \mathbb{R}_{+}$and $n \in \mathbb{Z}_{+}$, then the corresponding equation for one-particle distribution function of the system in the external field $\mathrm{V}_{0}(x)$ is the following:

$$
\frac{\partial}{\partial t} f_{1}(z ; t)+\left\langle p / m \mid \nabla_{x} f_{1}(z ; t)\right\rangle+\left\langle\nabla \mathrm{V}_{0}(x) \mid \nabla_{p} f_{1}(z ; t)\right\rangle=J\left(f_{1}(z ; t)\right),
$$


where $J\left(f_{1}(z ; t)\right)$ is the so-called "collision integral" [1-5] and is called the kinetic Boltzmann equation [1,3]. Below we will focus on the such special solutions of the Bogolubov's hierarchy of the Equations (72), using the developed above algebraic method of the Bogolubov's generating functional.

\section{Kinetic Boltzmann Equation in Non-Equilibrium Statistical Mechanics within Bogolubov's Approach}

4.1. Main Bogolubov Functional Equation and Its Solutions

Let us consider the Bogolubov's functional Equation (69) with the given initial data

$$
\begin{aligned}
& \frac{d}{d t} \mathcal{L}(f)=\int_{T^{*}(\Lambda)} d z\left\{\frac{1}{i} \frac{\delta \mathcal{L}(f)}{\delta f(z)}, T(p)\right\}^{(1)}+ \\
& +\int_{T^{*}(\Lambda)} d z_{1} \int_{T^{*}(\Lambda)} d z_{2}\left\{: \frac{1}{i} \frac{\delta}{\delta f\left(z_{1}\right)} \frac{1}{i} \frac{\delta}{\delta f\left(z_{2}\right)}: \mathcal{L}(f), \mathrm{V}\left(x_{1}-x_{2}\right)\right\}^{(2)}, \\
& \left.\mathcal{L}(f)\right|_{t=t_{0}}=\left.\operatorname{Tr}\left(P(t), t_{0}\right) \exp (i w(f))\right|_{\substack{t=t_{0} \\
\hbar \rightarrow 0}}=\mathcal{L}_{0}(f)
\end{aligned}
$$

where for all $t, t_{0} \in \mathbb{R}$

$$
P\left(t, t_{0}\right)=\exp \left[\frac{i}{\hbar}\left(t_{0}-t\right) \mathbb{H}\right] \bar{P} \exp \left[\frac{i}{\hbar}\left(t-t_{0}\right) \mathbb{H}\right]
$$

is a solution of the Heisenberg evolution equation

$$
\frac{d P}{d t}=\frac{i}{\hbar}[P, H],\left.\quad P\right|_{t=t_{0}}=\bar{P}
$$

for the statistical Gibbs operator $P: \Phi_{\mu} \rightarrow \Phi_{\mu}$ with $\operatorname{Tr} \bar{P}=1$.

When $\hbar \rightarrow 0$ in the Wigner representation the expression (78), as an explicit solution of the (79), allows the following expansion:

$$
\begin{aligned}
\mathcal{L}(f) & =\operatorname{Tr}\left(\exp \left[\frac{i}{\hbar}\left(t_{0}-t\right) \mathbb{H}\right] \bar{P} \exp \left[\frac{i}{\hbar}\left(t-t_{0}\right) \mathbb{H}\right] \exp [i w(f)]\right)= \\
& =\left.\operatorname{Tr}\left(\exp \left[\frac{i}{\hbar}\left(t_{0}-t\right)\left(\mathbb{H}_{0}+\mathrm{V}\right)\right] \bar{P} \exp \left[\frac{i}{\hbar}\left(t-t_{0}\right)\left(\mathbb{H}_{0}+\mathrm{V}\right)\right] \exp [i w(f)]\right)\right|_{\hbar \rightarrow 0}= \\
& =\operatorname{Tr}\left(\exp \left[\frac{i}{\hbar}\left(t_{0}-t\right) \mathbb{H}_{0}\right] \bar{P} \exp \left[\frac{i}{\hbar}\left(t-t_{0}\right) \mathbb{H}_{0}\right] \exp \left[\pi\left(t, t_{0}\right)\right] \exp [i w(f)]\right),
\end{aligned}
$$

where we marked $\mathbb{H}=\mathbb{H}_{0}+\mathrm{V}$,

$$
\begin{aligned}
& \mathbb{H}_{0}=\int_{T^{*}(\Lambda)} d z \frac{p^{2}}{2 m} w(z), \mathrm{V}=\int_{T^{*}(\Lambda)} d z_{1} \int_{T^{*}(\Lambda)} d z_{2} \Phi\left(x_{1}-x_{2}\right): w\left(z_{1}\right) w\left(z_{2}\right): \\
& \exp \left[\pi\left(t, t_{0}\right)\right]=P_{0}\left(t_{0}, t\right) P\left(t, t_{0}\right), \quad P_{0}\left(t_{0}, t\right)=\exp \left[\frac{i}{\hbar}\left(t_{0}-t\right) \mathbb{H}_{0}\right] \bar{P} \exp \left[\frac{i}{\hbar}\left(t-t_{0}\right) \mathbb{H}_{0}\right]
\end{aligned}
$$

Operator $\pi\left(t, t_{0}\right), t, t_{0} \in \mathbb{R}$, in (81) is called "cluster operator" and allows the next expansion into the unlimited series:

$$
\begin{aligned}
\pi\left(t_{0}, t\right) & =\sum_{n \in \mathbb{Z}_{+}} \frac{1}{n !} \int_{T^{*}(\Lambda)} d z_{1} \ldots \int_{T^{*}(\Lambda)} d z_{n} \pi_{n}\left(z_{1}, \ldots, z_{n} ; t, t_{0}\right) \times \\
\times & : \quad w\left(z_{1}\right) \ldots w\left(z_{n}\right): \stackrel{\text { def }}{=} \pi\left(t, t_{0} ; w\right),
\end{aligned}
$$


where the functions $\pi_{n}\left(z_{1}, \ldots, z_{n} ; t, t_{0}\right), n \in \mathbb{Z}_{+}$, can be defined uniquely form the representation (81) under condition that Gibbs operator $\bar{P}: \Phi_{\mu} \rightarrow \Phi_{\mu}$ is defined explicitly in the Wigner representation.

Thus, from (80)-(82) we obtain the following expressions for the Bogolubov's generating functional

$$
\begin{aligned}
\mathcal{L}(\mathrm{f}) & =\left.\operatorname{Tr}\left(P_{0} \exp \left[\pi\left(t, t_{0} ; w\right)\right] \exp (i w)\right)\right|_{\hbar \rightarrow 0}= \\
& =\exp \left[\pi\left(t, t_{0} ; \frac{1}{i} \frac{\delta}{\delta \mathrm{f}}\right)\right] \operatorname{Tr}\left(P_{0} \exp [i w(\mathrm{f})]\right)=\exp \left[\pi\left(t, t_{0} ; \frac{1}{i} \frac{\delta}{\delta \mathrm{f}}\right)\right] \mathcal{L}_{0}(\mathrm{f}),
\end{aligned}
$$

where $\mathcal{L}_{0}(\mathrm{f}), \mathrm{f} \in \mathcal{J}\left(T^{*}(\Lambda) ; \mathbb{R}\right)$, is a generating functional if the initial dynamical system of the particles under absent of interaction, that is

$$
\begin{gathered}
\mathcal{L}_{0}(\mathrm{f})\left(t, t_{0}\right)=\sum_{n \in \mathbb{Z}_{+}} \frac{1}{n !} \int_{T^{*}(\Lambda)} d z_{1} \ldots \int_{T^{*}(\Lambda)} d z_{n} \times \\
\times f_{n}\left(x_{1}+\frac{p_{1}}{m}\left(t_{0}-t\right), p_{1} ; \ldots ; x_{n}+\frac{p_{n}}{m}\left(t_{0}-t\right), p_{1}\right) \prod_{j=1}^{n}\left\{\exp \left[i f\left(z_{j}\right)\right]-1\right\} .
\end{gathered}
$$

Applying to (84), when $t_{0} \rightarrow-\infty$ the Bogolubov's correlation weakening (74), we obtain that for all $t \in \mathbb{R}_{+}$

$$
\mathcal{L}_{0}(f)(t)=\exp \left[\int_{T^{*}(\Lambda)} d z f_{1}\left(x-\frac{p}{m} t ; p\right)\{\exp [i f(z)]-1\}\right],
$$

where $\mathcal{L}_{0}(f)(t)=\lim _{t_{0} \rightarrow-\infty} \mathcal{L}_{0}(f)\left(t, t_{0}\right)$.

Thus, according to (83) and (85), we find that

$$
\mathcal{L}(f)(t)=\exp \left[\pi\left(t, t_{0} ; \frac{1}{i} \frac{\delta}{\delta f}\right) \mathcal{L}_{0}(f)(t)\right]
$$

is a solution of the Bogolubov's functional Equation (77), where

$$
\pi\left(t ; \frac{1}{i} \frac{\delta}{\delta f}\right)=\lim _{t_{0} \rightarrow-\infty} \pi\left(t, t_{0} ; \frac{1}{i} \frac{\delta}{\delta t}\right)
$$

for all $t \in \mathbb{R}_{+}$. To specify the form of the operators (87) we note, that operator $\xi\left(t, t_{0} ; w\right)=$ $\exp \left[\pi\left(t, t_{0} ; w\right)\right]$ for all $t, t_{0} \in \mathbb{R}_{+}$satisfies under $\hbar \rightarrow 0$ the following differential evolution relationship:

$$
\frac{d \xi}{d t}=\frac{1}{i \hbar}[\xi, \mathbb{H}]_{0}+\lim _{\hbar \rightarrow 0} \frac{1}{\hbar}\left(\mathrm{V}-P_{0}^{-1} \mathrm{~V} P_{0}\right) \xi,
$$

where all operators assumed to be given in the Wigner representation. Expanding operator $\xi\left(t, t_{0}, \mathcal{W}\right)$ into the sum of $n$-particles components, $n \in \mathbb{Z}_{+}$, we find

$$
\xi\left(t, t_{0} ; w\right)=\sum_{n \in \mathbb{Z}_{+}} \frac{1}{n !} \int_{T^{*}(\Lambda)} d z_{1} \ldots \int_{T^{*}(\Lambda)} d z_{n} \xi_{n}\left(z_{1}, \ldots, z_{n} ; t, t_{0}\right): w\left(z_{1}\right) \ldots w\left(z_{n}\right):
$$


and there is mutually unambiguous correspondence $[3,7]$ between coefficient functions $\xi_{n}\left(z_{1}, \ldots, z_{n} ; t, t_{0}\right)$ in (89) and coefficient functions in the expansion (82)

$$
\begin{aligned}
& \pi_{n}\left(z_{1}, \ldots, z_{n}\right)=\sum_{\sigma \in \Sigma_{n}}(-1)^{n+i}(\sigma-1) \prod_{j=1}^{\infty} \xi_{\sigma}\left(z_{\langle k\rangle} \in \sigma_{j}\right), \\
& \xi_{n}\left(z_{1}, \ldots, z_{n}\right)=\sum_{\sigma \in \Sigma_{n}} \prod_{j=1}^{\infty} \pi_{\sigma_{j}}\left(z_{\langle k\rangle} \in \sigma_{j}\right) .
\end{aligned}
$$

Here, $\sigma \in \Sigma_{n}$ is an arbitrary partition of the symmetry group $\Sigma_{n}$ of all permutations of the set of numbers $\{1,2, \ldots, n\}$ on the subsets $\left\{\sigma_{j}: j=1, \ldots, s\right\}$, which are not intersect, that is $\bigcup_{j=1}^{n} \sigma_{j}=\{1, \ldots, n\}$ and $\xi_{\sigma_{j}}$ and $\pi_{\sigma_{j}}, j=1, \ldots, s$, are the corresponding to this partition coefficient functions. In particular,

$$
\xi_{1}\left(z_{1}\right)=\pi_{1}\left(z_{1}\right), \quad \pi_{2}\left(z_{1}, z_{2}\right)=\xi_{2}\left(z_{1}, z_{2}\right)-\xi_{1}\left(z_{1}\right) \xi_{1}\left(z_{2}\right)
$$

and so on. Thus, on the base of defined operator series (82) or (89) the problem of the explicit calculations of the distribution functions become very simple. Below we will analyze these series by means of the language of the Bogolubov's generating functional $\mathcal{L}(f), f \in \mathcal{J}\left(T^{*}(\Lambda) ; \mathbb{R}\right)$, using the Bogolubov's functional hypothesis [1-5].

\subsection{Bogolubov-Boltzmann Kinetic Equation in the Frame of Functional Hypothesis}

The generating functional, as it was stated above, is given by the expression

$$
\mathcal{L}(f)(t)=\exp \left[\pi\left(t_{0} ; \frac{1}{i} \frac{\delta}{\delta f}\right)\right] \mathcal{L}_{0}(f)(t) .
$$

Here, $\mathcal{L}_{0}(f)(t), t \in \mathbb{R}_{+}$, is a generating functional of the system of non-interacting particles, which is equal to the expression (85) when $t_{0} \rightarrow-\infty$. From (91) follows, that for all $t \in \mathbb{R}_{+}$for the $n$-particle distribution function $f_{n}\left(z_{1}, \ldots, z_{n} ; t\right)$ the general functional relationship holds:

$$
f_{n}\left(z_{1}, \ldots, z_{n} ; t\right)=f_{n}\left(z_{1}, \ldots, z_{n} ; f_{1}(z ; t)\right) .
$$

Respectively, the generating functional (91) satisfies, according to (68) when $t=0$, the following dynamic equation:

$$
\begin{aligned}
& \frac{d}{d t} \mathcal{L}(\mathrm{f})=\int_{T^{*}(\Lambda)} d z\left\{\frac{1}{i} \frac{\delta \mathcal{L}(\mathrm{f})}{\delta \mathrm{f}(z)}, T(p)\right\}^{(1)}+ \\
& +\frac{1}{2} \int_{T^{*}(\Lambda)} d z_{1} \int_{T^{*}(\Lambda)} d z_{2}\left\{: \frac{1}{i} \frac{\delta}{\delta \mathrm{f}\left(z_{1}\right)} \frac{1}{i} \frac{\delta}{\delta \mathrm{f}\left(z_{2}\right)}: \mathcal{L}(\mathrm{f}), \mathrm{V}\left(x_{1}-x_{2}\right)\right\}^{(2)},
\end{aligned}
$$

Let us put $f_{1}(z) \rightarrow f_{1}(z ; \tau)$, where $\tau \in \mathbb{R}_{-}$and

$$
\frac{\partial f_{1}(z ; \tau)}{\partial \tau}=\left\{f_{1}(z ; \tau), T(p)\right\}^{(1)}+\int_{T^{*}(\Lambda)} d z_{1}\left\{f_{1}\left(z_{1}\right) f_{1}(z), \mathrm{V}\left(x_{1}-x\right)\right\}^{(2)} .
$$

Then from (93) we also obtain that

$$
\frac{d}{d \tau} \mathcal{L}(\mathrm{f})=\{\{\mathcal{L}(\mathrm{f}), \mathcal{H}(\mathcal{F})\}\}=\int_{T^{*}(\Lambda)} d z\left\{\frac{1}{i} \frac{\delta \mathcal{L}(\mathrm{f})}{\delta \mathrm{f}(z)}, T(p)+\int_{T^{*}(\Lambda)} d z_{1} f_{1}\left(z_{1}\right), \mathrm{V}\left(x_{1}-x\right)\right\}^{(1)}
$$


for all $\tau \in \mathbb{R}_{-}$. Then Equation (95) can be rewritten in the following way:

$$
\frac{d}{d \tau} \mathcal{L}(\mathrm{f})=\{\{\mathcal{L}(\mathrm{f}), \tilde{\mathcal{H}}(\mathcal{F})\}\}=\int_{T^{*}(\Lambda)} d z\left\{\frac{1}{i} \frac{\delta \mathcal{L}(\mathrm{f})}{\delta \mathrm{f}(z)}, \tilde{H}\left(f_{1}\right)\right\}^{(1)}
$$

where, by definition, $\tilde{H}\left(f_{1}\right):=\frac{\delta}{\delta f_{1}} \tilde{\mathcal{H}}(\mathcal{F})$ and

$$
\tilde{\mathcal{H}}(\mathcal{F})=\int_{T^{*}(\Lambda)} d z \frac{p^{2}}{2 m} f_{1}(z)+\frac{1}{2} \int_{T^{*}(\Lambda)} d z_{1} \int_{T^{*}(\Lambda)} d z_{2} f_{1}\left(z_{1}\right) f_{1}\left(z_{2}\right) \mathrm{V}\left(x_{1}-x_{2}\right),
$$

is the Vlasov-type Hamiltonian of the self-consistent particles interaction. Let us define the following mapping on the phase space of $n \in \mathbb{Z}_{+}$particles:

$$
S_{n}(\tau) x_{j}=x_{j}(\tau), \quad S_{n}(\tau) p_{j}=p_{j}(\tau),
$$

where for all $\tau \in \mathbb{R}_{-}, j=\overline{1, n}$,

$$
\begin{aligned}
& \frac{d x(\tau)}{d t}=\{\tilde{H}, x(\tau)\}^{(1)}, \frac{d p(\tau)}{d t}=\{\tilde{H}, p(\tau)\}^{(1)}, \\
& \tilde{H}=\sum_{j=1}^{n} \frac{p_{j}^{2}}{2 m}+\frac{1}{2} \sum_{j=k}^{n} \mathrm{~V}\left(x_{j}-x_{k}\right) .
\end{aligned}
$$

It easy to see, that system of Equations (99) gives the exact solution [32] for the dual Equation (94) in the form of the sum of $\delta$-functions of $n \in \mathbb{N}$ particles:

$$
f_{1}(z)=\sum_{j=1}^{n} \delta\left(z-z_{j}\right)
$$

where $z_{j} \in \Lambda, j=1, \ldots, n$, are the coordinates of the cluster. Using (98) from (96) we obtain, that for all $\tau \in \mathbb{R}$

$$
\begin{aligned}
& \frac{d}{d \tau} \mathcal{L}(\mathrm{f})(\tau)=\{\{\mathcal{L}(\mathrm{f})(\tau), \tilde{\mathcal{H}}(\mathcal{F})\}\}+\frac{1}{2} \int_{\mathbb{R}^{3} \times \mathbb{R}^{3}} d z_{1} \int_{T^{*}(\Lambda)} d z_{2} \times \\
& \times\left\{: \frac{1}{i} \frac{\delta}{\delta \mathrm{f}\left(z_{1}\right)} \frac{1}{i} \frac{\delta}{\delta \mathrm{f}\left(z_{2}\right)}: \mathcal{L}(\mathrm{f}), \overline{\mathrm{V}}\left(x_{1}-x_{2}\right)(\tau)\right\}^{(2)},
\end{aligned}
$$

where we denoted

$$
\begin{aligned}
& \mathcal{L}(\mathrm{f})(\tau)=S(\tau) \mathcal{L}\left(\mathrm{f} \mid S(-\tau) f_{1}\right) \\
& \overline{\mathrm{V}}\left(x_{1}-x_{2}\right)(\tau)=S(\tau) \mathrm{V}\left(S(-\tau)\left(x_{1}-x_{2}\right)\right) \\
& f_{2}\left(z, z_{1}\right)(\tau)=S(\tau) f_{2}\left(z_{1}, z \mid S(-\tau) f_{1}\right)
\end{aligned}
$$

Integrating the Equation (101) in limits $\tau \in(-\infty, 0)$, we obtain, that

$$
\begin{aligned}
& \left.\mathcal{L}(\mathrm{f})\right|_{\tau=0}=\lim _{\tau \rightarrow-\infty} S(\tau) \mathcal{L}\left(\mathrm{f} \mid S(-\tau) f_{1}\right)+\int_{-\infty}^{0} d \tau\left\{\frac{1}{i} \frac{\delta}{\delta \mathrm{f}(z)} \mathcal{L}(\mathrm{f})(\tau), \tilde{\mathcal{H}}(\mathcal{F}(\tau))\right\}^{(1)}+ \\
& \left.\quad+\frac{1}{2} \int_{-\infty}^{0} d \tau \int_{\mathbb{R}^{3} \times \mathbb{R}^{3}} d z_{1} \int_{T^{*}(\Lambda)} d z_{2}\left\{: \frac{1}{i} \frac{\delta}{\delta \mathrm{f}\left(z_{1}\right)} \frac{1}{i} \frac{\delta}{\delta \mathrm{f}\left(z_{2}\right)}: \mathcal{L}(\mathrm{f}), \overline{\mathrm{V}}\left(x_{1}-x_{2}\right)(\tau)\right\}^{(2)}\right]
\end{aligned}
$$


We should also note here, that due to the Bogolubov's principle of correlations attenuation (74) and using (85) the first item in (103) can be represented in the form

$$
\begin{gathered}
\lim _{\tau \rightarrow-\infty} S(\tau) \mathcal{L}\left(\mathrm{f} \mid S(-\tau) f_{1}\right)=\lim _{\tau \rightarrow \infty} \exp \left[\int_{T^{*}(\Lambda)} d z S(\tau) f_{1}(z)(\tau)\{\exp [i \mathrm{f}(z)]-1\}\right]= \\
=\exp \left[\int_{T^{*}(\Lambda)} d z f_{1}(z)\{\exp [i \mathrm{f}(z)]-1\}\right] .
\end{gathered}
$$

Applying to the expression (103) the different variants of the successive approximations method [1-5], we can get the generating functional $\mathcal{L}(f)$ in explicit form and then, using formula

$$
f_{n}\left(z_{1}, \ldots, z_{n}\right)=: \frac{1}{i} \frac{\delta}{\delta f\left(z_{1}\right)} \ldots \frac{1}{i} \frac{\delta}{\delta f\left(z_{n}\right)}:\left.\mathcal{L}(f)\right|_{f=0}
$$

for all $n \in \mathbb{Z}_{+}$obtain distribution function for any order of perturbation theory. In particular, choosing expansion by the particle density in container $A \in \Lambda$ as a small parameter, it is easy to get modified kinetic Bogolubov-Boltzmann equation for oneparticle distribution function $f_{1}(z ; t), z \in T^{*}(\Lambda), t \in \mathbb{R}_{+}$:

$$
\frac{\partial f_{1}\left(z_{1} ; t\right)}{\partial t}+\left\langle\frac{p}{m} \mid \nabla_{x} f_{1}\left(z_{1} ; t\right)\right\rangle=\int_{T^{*}(\Lambda)} d z_{2}\left\{\tilde{f}_{2}\left(z_{1}, z_{2} ; t\right), \mathrm{V}\left(x_{1}-x_{2}\right)\right\}^{(2)},
$$

where the function $\tilde{f}_{2}\left(z_{2}, z_{1} ; t\right)$ is defined according to (103) and (104) by the following expression:

$$
\begin{aligned}
& \tilde{f}_{2}\left(z_{2}, z_{1} ; t\right)=f_{1}\left(\tilde{z}_{2} ; t\right) f_{1}\left(\tilde{z}_{1} ; t\right), \\
& \tilde{z}_{j}=\lim _{\tau \rightarrow \infty} S_{2}(\tau) S_{1}(-\tau) z_{j} \Rightarrow\left\{\begin{array}{l}
\tilde{x}_{j}=\lim _{\tau \rightarrow \infty} S_{2}(-\tau) x_{j}+\tau \frac{p_{j}}{m}, \\
\tilde{p}_{j}=\lim _{\tau \rightarrow \infty} S_{2}(-\tau) p_{j},
\end{array}\right.
\end{aligned}
$$

for $j=1$,2. Taking into account, that Poisson bracket $\{\cdot, \cdot\}^{(n)}$ is invariant with respect to the mappings $S_{n}(\tau), \tau \in \mathbb{Z}_{+}$, from (107) it is easy to find, that

$$
\begin{gathered}
\left\{\tilde{f}_{2}\left(z_{1}, z_{2} ; t\right), \mathrm{V}\left(x_{2}-x_{1}\right)\right\}^{(2)}=\frac{\left|p_{2}-p_{1}\right|}{m} \frac{\partial}{\partial \xi}\left(f_{1}\left(\tilde{z}_{1} ; t\right) f_{1}\left(\tilde{z}_{2} ; t\right)\right)- \\
-\left\langle\frac{\left(\tilde{p}_{2}-p_{1}\right)}{m} \mid \nabla_{x_{1}} f_{1}\left(\tilde{z}_{1} ; t\right)\right\rangle f_{1}\left(\tilde{z}_{2} ; t\right)+\left\langle\frac{\left(\tilde{p}_{2}-p_{1}\right)}{m} \mid \nabla_{x_{2}} f_{1}\left(\tilde{z}_{2} ; t\right)\right\rangle f_{1}\left(\tilde{z}_{1} ; t\right),
\end{gathered}
$$

where $\xi \in \mathbb{R}^{1}$ is a parameter of the axis in cylindrical coordination system which directed along the vector $\left(p_{2}-p_{1}\right) \in \mathbb{E}^{3}$ and with beginning at the point $x_{1} \in \Lambda$. After substituting (108) into (106) we can get kinetic Bogolubov-Boltzmann equation [1-5] in form of (76) with the explicitly defined collision integral $J\left(f_{1}\right)$, obtained from (108) via integration by $\xi \in \mathbb{R}$. Choosing in (104) other approximations of the generating functional $\mathcal{L}(f), f \in \mathcal{J}\left(T^{*}(\Lambda) ; \mathbb{R}\right)$, one can find other forms of Bogolubov-Boltzmann kinetic Equation (76).

We can also make a remark concerning the nature of the operator-functional expression (87) or (82). Namely, it is easy to see that generating functional $\mathcal{L}(f)\left(t, t_{0}\right)$, $\mathrm{f} \in \mathcal{J}\left(T^{*}(\Lambda) ; \mathbb{R}\right)$, allows the following operator-functional representation for all $t, t_{0} \in \mathbb{R}$ :

$$
\begin{aligned}
\mathcal{L}(\mathrm{f})\left(t, t_{0}\right) & =\exp \left[\frac { 1 } { 2 } \int _ { T ^ { * } ( \Lambda ) } d z _ { 1 } \int _ { T ^ { * } ( \Lambda ) } d z _ { 2 } \left\{: \frac{1}{i} \frac{\delta}{\delta \mathrm{f}\left(z_{1}\right)} \times\right.\right. \\
& \left.\left.\times \frac{1}{i} \frac{\delta}{\delta \mathrm{f}\left(z_{2}\right)}:, \mathrm{V}\left(x_{1}-x_{2}\right)\right\}^{(2)}\left(t-t_{0}\right)\right] \mathcal{L}_{0}(\mathrm{f})\left(t, t_{0}\right) .
\end{aligned}
$$


Comparing the expressions (109) and (83), we find that, for arbitrary $t, t_{0}$,

$$
\begin{gathered}
\pi\left(t, t_{0} ; \frac{1}{i} \frac{\delta}{\delta f}\right)=\frac{1}{2}\left(t-t_{0}\right) \times \\
\times \int_{T^{*}(\Lambda)} d z_{1} \int_{T^{*}(\Lambda)} d z_{2}\left\{: \frac{1}{i} \frac{\delta}{\delta f\left(z_{1}\right)} \frac{1}{i} \frac{\delta}{\delta f\left(z_{2}\right)}:, \mathrm{V}\left(x_{1}-x_{2}\right)\right\}^{(2)}
\end{gathered}
$$

as the functional $\mathcal{L}_{0}(f)\left(t, t_{0}\right), f \in \mathcal{J}\left(T^{*}(\Lambda) ; \mathbb{R}\right)$, is arbitrary. It is easy to see from (110), that operator $\pi\left(t, t_{0} ; \frac{1}{i} \frac{\delta}{\delta f}\right)$ is not poly-local with respect to the functional derivatives $\frac{1}{i} \frac{\delta}{\delta f}$, which corresponds to the singularity in the operator expansion (82). Thus, using the expression (109), arbitrariness of the initial state and Bogolubov's attenuation correlation condition gives a possibility to find many types of the solutions via the method of successive approximations, which follows from (109) and Bogolubov's functional hypothesis.

\section{Kinetic Equations, Their Algebraic Structure and Invariant Reductions}

\subsection{Lie-Algebraic Setting}

It is well known that the classical Bogolubov-Boltzmann kinetic equations under the condition of many-particle correlations $[1,3-6,9,19,32,34,36-38]$ at weak short range interaction potentials describe long waves in a dense gas medium. The same equation, called the Vlasov one, as it was shown by N. Bogolubov [32], describes also exact microscopic solutions of the infinite Bogolubov chain [34] for the many-particle distribution functions, which was widely studied making use of both classical approaches in $[3,19,20,38-48]$ and in $[10,11,15,49-54]$, making use of the generating Bogolubov functional method and the related quantum current algebra representations.

A.A. Vlasov proposed his kinetic equation [18] for electron-ion plasma, based on general physical reasonings, that in contrast to the short range interaction forces between neutral gas atoms, interaction forces between charged particles slowly decrease with distance, and therefore the motion of each such particle is determined not only by its pairwise interaction with either particle, yet also by the interaction with the whole ensemble of charged particles. In this case the Bogolubov equation for distribution functions in a domain $\Lambda \subset \mathbb{R}^{3}$

$$
\frac{\partial f_{1}(z ; t)}{\partial t}+\left\langle\frac{p}{m} \mid \nabla_{x} f_{1}(z ; t)\right\rangle=\int_{T^{*}(\Lambda)} d z^{\prime}\left\{f_{2}\left(z, z^{\prime} ; t\right), V\left(x-x^{\prime}\right)\right\}^{(2)},
$$

where $z:=(x, p) \in T^{*}(\Lambda), t \in \mathbb{R}_{+}$is the temporal evolution parameter, $\{\cdot, \cdot\}^{(m)}$ denotes the canonical Poisson bracket $[3,21,22]$ on the product $T^{*}(\Lambda)^{m}, m \in \mathbb{N}$, and $V\left(x-x^{\prime}\right), x, x^{\prime} \in \Lambda$, is an interparticle interaction potential, reduces to the Vlasov equation if to put in (111)

$$
f_{2}\left(z, z^{\prime} ; t\right)=f_{1}(z ; t) f_{1}\left(z^{\prime} ; t\right)
$$

that is to assume that the two-particle correlation function $[19,20,36,38]$ vanishes:

$$
g_{2}\left(z, z^{\prime} ; t\right)=f_{2}\left(z, z^{\prime} ; t\right)-f_{1}(z ; t) f_{1}\left(z^{\prime} ; t\right)=0
$$

for all $z, z^{\prime} \in T^{*}(\Lambda)$ and $t \in \mathbb{R}_{+}$. Then, one easily obtains from (111) that

$$
\frac{\partial f_{1}(z ; t)}{\partial t}+\left\langle\frac{p}{m} \mid \nabla_{x} f_{1}(z ; t)\right\rangle=\left\langle\frac{\partial f_{1}(z ; t)}{\partial p} \mid \nabla_{x} \int_{T^{*}(\Lambda)} d z^{\prime} V\left(x-x^{\prime}\right) f_{1}\left(z^{\prime} ; t\right)\right\rangle
$$

for all $z \in T^{*}(\Lambda)$ and $t \in \mathbb{R}_{+}$. Remark here that the Equation (114) is reversible under the time reflection $\mathbb{R}_{-} \ni-t \rightleftarrows t \in \mathbb{R}_{+}$, thus it is obvious that it can not describe thermodynamically stable limiting states of the particle system in contrast to the classical Bogolubov-Boltzmann kinetic equations $[1,3,6,10,15,19,34,38]$, being a priori time non- 
reversible owing to the choice of boundary conditions in the correlation weakening form. This means that in spite of the Hamiltonicity of the Bogolubov chain for the distribution functions, the Bogolubov-Boltzmann equation a priori is not reversible. It is also evident that the condition (113) does not break the Hamiltonicity-the Equation (114) is Hamiltonian with respect to the following Lie-Poisson-Vlasov bracket:

$$
\{\{a(f), b(f)\}\}:=\int_{T^{*}(\Lambda)} d z f(z)\{\operatorname{grad} a(f)(z), \operatorname{grad} b(f)(z)\}^{(1)},
$$

where $\operatorname{grad}(\cdot):=\delta(\cdot) / \delta f, f \in D\left(T^{*}(\Lambda)\right):=M_{f_{1}}$, respectively $a, b \in D\left(M_{f_{1}}\right)$ are smooth functionals on the functional manifold $M_{f_{1}}$, consisting of functions fast decreasing at the boundary $\partial \Lambda$ of the domain $\Lambda \subset \mathbb{R}^{3}$. The statement above easily ensues from the following proposition.

Proposition 2. Let $M_{\mathcal{F}}$ denote a set of many-particle distribution functions. Then the classical Bogolubov-Poisson bracket $[10,11,34,44]$ on the functional space $D\left(M_{\mathcal{F}}\right)$ reduces invariantly on the subspace $D\left(M_{f_{1}}\right) \subset D\left(M_{\mathcal{F}}\right)$ to the Lie-Poisson-Vlasov bracket (115).

This proposition and its different consequences will be analyzed in more detail in sections to follow below.

\subsection{The Lie-Poisson-Vlasov Bracket}

The bracket expression (115) allows a slightly different Lie-algebraic interpretation, based on considering the functional space $D\left(M_{f_{1}}\right)$ as a Poissonian manifold, related with the canonical symplectic structure on the diffeomorphism group $\operatorname{Diff}(\Lambda)$ of the domain $\Lambda \subset \mathbb{R}^{3}$, first described $[55,56]$ still in 1887 by Sophus Lie. Namely, the following classical theorem holds.

Theorem 4. The Lie-Poisson bracket at point $(\mu ; \eta) \in T_{\eta}^{*}(\operatorname{Dif} f(\Lambda))$ on the co-adjoint space $T_{\eta}^{*}(\operatorname{Dif} f(\Lambda)), \eta \in \operatorname{Diff}(\Lambda)$, is equal to the expression

$$
\{f, g\}(\mu)=(\mu \mid[\delta g(\mu) / \delta \mu, \delta f(\mu) / \delta \mu])_{c}
$$

for any smooth right-invariant functionals $f, g \in C^{\infty}\left(T_{\eta}^{*}(\operatorname{Diff}(\Lambda)) ; \mathbb{R}\right)$.

Proof. By classical definition [21,22,55-57] of the Poisson bracket of smooth functions $(\mu \mid a)_{c},(\mu \mid b)_{c} \in C^{\infty}\left(T_{\eta}^{*}(\operatorname{Diff}(\Lambda)) ; \mathbb{R}\right), a, b \in \operatorname{diff}(\Lambda) \simeq T_{\eta}(\operatorname{Diff}(\Lambda))$ on the symplectic space $T_{\eta}^{*}(\operatorname{Diff}(\Lambda))$, it is easy to calculate that

$$
\begin{gathered}
\{\mu(a), \mu(b)\}:=\delta \alpha\left(X_{a}, X_{b}\right)= \\
=X_{a}\left(\alpha \mid X_{b}\right)_{c}-X_{b}\left(\alpha \mid X_{a}\right)_{c}-\left(\alpha \mid\left[X_{a}, X_{b}\right]\right)_{c}
\end{gathered}
$$

where $X_{a}:=\delta(\mu \mid a)_{c} / \delta \mu=a \in \operatorname{diff}(\Lambda), X_{b}:=\delta(\mu \mid b)_{c} / \delta \mu=b \in \operatorname{diff}(\Lambda)$. Since the expressions $X_{a}\left(\alpha \mid X_{b}\right)_{c}=0$ and $X_{b}\left(\alpha \mid X_{a}\right)_{c}=0$ owing the right-invariance of the vector fields $X_{a}, X_{b} \in T_{\eta}(\operatorname{Diff}(\Lambda))$, the Poisson bracket (117) transforms into

$$
\begin{gathered}
\left\{(\mu \mid a)_{c},(\mu \mid b)_{c}\right\}=-\left(\alpha \mid\left[X_{a}, X_{b}\right]\right)_{c}= \\
=(\mu \mid[b, a])_{c}=\left(\mu \mid\left[\delta(\mu \mid b)_{c} / \delta \mu, \delta(\mu \mid a)_{c} / \delta \mu\right]\right)_{c}
\end{gathered}
$$

for all $(\mu ; \eta) \in T_{\eta}^{*}(\operatorname{Diff}(\Lambda)) \simeq \operatorname{diff}^{*}(\Lambda)$, and any $a, b \in \operatorname{diff}(\Lambda)$. The Poisson bracket (118) is easily generalized to

$$
\{f, g\}(\mu)=(\mu \mid[\delta g(\mu) / \delta \mu, \delta f(\mu) / \delta \mu])_{c}
$$

for any smooth functionals $f, g \in C^{\infty}\left(\operatorname{diff} f^{*}(\Lambda) ; \mathbb{R}\right)$, finishing the proof. 
Concerning our special problem of describing evolution equations for one-particle distribution functions, we will consider the one particle cotangent space $T^{*}(\Lambda)$ over a domain $\Lambda \subset \mathbb{R}^{3}$ and the canonical Poisson bracket $\{\cdot, \cdot\}:=\{\cdot, \cdot\}^{(1)}$ on $T^{*}(\Lambda)$, for which, by definition, for any $f, g \in M_{f_{1}}$

$$
\{f, g\}(z):=\left\langle\frac{\partial f}{\partial p} \mid \frac{\partial g}{\partial x}\right\rangle-\left\langle\frac{\partial g}{\partial p} \mid \frac{\partial f}{\partial x}\right\rangle,
$$

where $z=(x, p) \in T^{*}(\Lambda)$. Denote now by $\mathcal{G}:=\left(M_{f_{1}} ;\{\cdot, \cdot\}\right)$ the related functional Lie algebra and $\mathcal{G}^{*}$ its adjoint space with respect to the standard bilinear symmetric form $(\cdot \mid \cdot): M_{f_{1}} \times M_{f_{1}} \rightarrow \mathbb{R}$ on the product $M_{f_{1}} \times M_{f_{1}}$, where

$$
(f \mid g):=\int_{T^{*}(\Lambda)} f(z) g(z) d z .
$$

The constructed Lie algebra $\mathcal{G}$ with respect to the bilinear symmetric form (121) proves to be metrized, that is $\mathcal{G} \simeq \mathcal{G}^{*}$ and

$$
(\{f, g\} \mid h)=(f \mid\{g \mid h\})
$$

for any $f, g$ and $h \in \mathcal{G}$. If $\gamma \in D\left(\mathcal{G}^{*}\right)$ is a smooth functional on $\mathcal{G}^{*}$, its gradient $\operatorname{grad} \gamma(f) \in$ $\mathcal{G}$ at point $f \in \mathcal{G}^{*}$ is naturally defined via the limiting expression

$$
(g \mid \operatorname{grad} \gamma(f)):=\left.\frac{d}{d \varepsilon} \gamma(f+\varepsilon g)\right|_{\varepsilon=0}
$$

for arbitrary element $g \in \mathcal{G}^{*}$. Define now the Poisson structure $\{\{\cdot, \cdot\}\}: \mathcal{G}^{*} \times \mathcal{G}^{*} \rightarrow \mathbb{R}$ by means of the standard Lie-Poisson $[7,21,22,37,55,56,58]$ expression:

$$
\{\{\gamma, \mu\}\}:=(f \mid\{\operatorname{grad} \gamma(f), \operatorname{grad} \gamma(f)\})
$$

for arbitrary functionals $\gamma, \mu \in D\left(\mathcal{G}^{*}\right)$. It is evident that the expression (124) identically coincides with the Poisson bracket (115).

Consider a functional $\gamma \in D\left(\mathcal{G}^{*}\right)$ and the related co-adjoint action of the element $\operatorname{grad} \gamma(f) \in \mathcal{G}$ at a fixed element $f:=f_{1} \in \mathcal{G}^{*}$ :

$$
\partial f_{1} / \partial t:=a d_{\operatorname{grad} \gamma\left(f_{1}\right)}^{*} f_{1},
$$

where $t \in \mathbb{R}$ is the corresponding evolution parameter. It is easy observe that

$$
\partial f_{1} / \partial t=\left\{\left\{\gamma, f_{1}\right\}\right\}
$$

is a Hamiltonian equation with the functional $\gamma \in D\left(\mathcal{G}^{*}\right)$ taken as its Hamiltonian, being simultaneously equivalent to the following canonical Hamiltonian flow:

$$
\partial f_{1} / \partial t=\left\{f_{1}, \operatorname{grad} \gamma\left(f_{1}\right)\right\},
$$

if to choose as a Hamiltonian the following functional

$$
\gamma\left(f_{1}\right):=\int_{T^{*}(\Lambda)} d z_{1} \frac{p_{1}^{2}}{2 m} f_{1}\left(z_{1}\right)+\frac{1}{2} \int_{T^{*}(\Lambda)^{2}} d z_{1} d z_{2} V\left(x_{1}-x_{2}\right) f_{1}\left(z_{1}\right) f_{1}\left(z_{2}\right),
$$

where $V\left(x_{1}-x_{2}\right)$ is a two-particle interaction potential, $x_{1}, x_{2} \in \Lambda$. It is easy to observe here that the Hamiltonian (128) is obtained from the corresponding classical Bogolubov Hamiltonian expression 


$$
\mathcal{H}(\mathcal{F}):=\int_{T^{*}(\Lambda)} d z_{1} \frac{p_{1}^{2}}{2 m} f_{1}\left(z_{1}\right)+\frac{1}{2} \int_{T^{*}(\Lambda)^{2}} d z_{1} d z_{2} V\left(x_{1}-x_{2}\right) f_{2}\left(z_{1}, z_{2}\right),
$$

where $\mathcal{F}=\left(f_{1}, f_{2}, \ldots\right) \in M_{\mathcal{F}}$ denotes an infinite vector from the space $M_{\mathcal{F}}:=\prod_{j \in \mathbb{N}} M_{f_{j}}$ of multiparticle distribution functions, and if to impose on it the constraint (112). Thus we have stated the following proposition.

Proposition 3. The Boltzmann-Vlasov kinetic Equation (114) is a Hamiltonian system on the functional manifold $\mathcal{G}^{*} \simeq \mathcal{G}=\left(M_{f} ;\{\cdot, \cdot\}\right)$ with respect to the canonical Lie-Poisson structure (124) with Hamiltonian (128). As a consequence, the flow (114) is time reversible.

\subsection{The Boltzmann-Vlasov Equation, Its Microscopic Exact Solutions and Functional Properties}

Proposition 2, stated above, claims that the Boltzmann-Vlasov Equation (114) is a suitable reduction of the whole Bogolubov chain upon the invariant functional subspace $M_{f_{1}} \subset M_{\mathcal{F}}$. Moreover, this invariance in no way should be compatible a priori $[10,11,15,32,45,46,50]$ with the other kinetic equations from the Bogolubov chain, and can be even contradictory. Nonetheless, as it was stated [32] by N. Bogolubov, namely owing to this invariance of the subspace $M_{f_{1}} \subset M_{\mathcal{F}}$ the Boltzmann-Vlasov Equation (114) in the case of the Boltzmann-Enskog hard sphere approximation of the interparticle potential possesses exact microscopical solutions which are compatible with the whole hierarchy of the Bogolubob kinetic equations. The latter is, obviously, equivalent to its Hamiltonicity on the manifold $M_{f_{1}}$ with respect to the Lie-Poisson bracket (124). The Boltzmann-Enskog kinetic equation $[6,20,32,36,38]$ equals

$$
\begin{gathered}
\frac{\partial f_{1}(z ; t)}{\partial t}+\left\langle\frac{p}{m} \mid \nabla_{x} f_{1}(z ; t)\right\rangle= \\
=a^{2} \int_{\mathbb{S}^{2}} d n \int_{\mathbb{E}^{3}} d p^{\prime}\left\langle p^{\prime} \mid n\right\rangle\left\langle\frac{\tilde{p}^{\prime}}{m} \mid n\right\rangle\left[f_{2}\left(x, \tilde{p} ; x+a n, \tilde{p}^{\prime} ; t\right)-f_{2}\left(x, p ; x-a n, p^{\prime} ; t\right)\right]
\end{gathered}
$$

where $\left.\tilde{p}:=p+n\left\langle p^{\prime}-p \mid n\right\rangle, \tilde{p}^{\prime}:=p-n\left\langle p^{\prime}-p \mid n\right\rangle, a\right\rangle 0-$ a particle diameter, $n \in \mathbb{S}^{2}$ - a unit vector, $\langle n \mid n\rangle=1$, and, by definition, $f_{2}\left(z, z^{\prime} ; t\right)=0$ for all $z, z^{\prime} \in T^{*}(\Lambda), t \in \mathbb{R}$, satisfying the condition $\left\|z-z^{\prime}\right\|\langle a$. The Equation (130) easily reduces to the Vlasov-Enskog equation

$$
\begin{gathered}
\frac{\partial f_{1}(z ; t)}{\partial t}+\left\langle\frac{p}{m} \mid \nabla_{x} f_{1}(z ; t)\right\rangle=J_{V-E}(f), \\
J_{V-E}(f)=a^{2} \int_{\mathbb{S}^{2}} d n \int_{\mathbb{E}^{3}} d p^{\prime}\left\langle p^{\prime} \mid n\right\rangle\left\langle\frac{\tilde{p}}{m}^{\prime} \mid n\right\rangle \times \\
\times\left[f_{1}(x, \tilde{p} ; t) f_{1}\left(x+a n, \tilde{p}^{\prime} ; t\right)-f_{1}(x, p ; t) f_{1}\left(x-a n, p^{\prime} ; t\right)\right]
\end{gathered}
$$

for all $(z ; t) \in T^{*}(\Lambda) \times \mathbb{R}$, owing to its Hamiltonicity on the space $M_{f_{1}} \subset M_{\mathcal{F}}$. If, in addition, there exists a nontrivial interparticle potential, the equation above is naturally generalized to the kinetic equation

$$
\begin{gathered}
\frac{\partial f_{1}(z ; t)}{\partial t}+\left\langle\frac{p}{m} \mid \nabla_{x} f_{1}(z ; t)\right\rangle=J_{V-E}(f)+ \\
+\int_{T^{*}(\Lambda)} d z^{\prime}\left\{f_{1}(z ; t) f_{1}\left(z^{\prime} ; t\right), V\left(x-x^{\prime}\right)\right\}^{(2),}
\end{gathered}
$$

which remains to be Hamiltonian on $M_{f_{1}}$ and possesses, in particular, the following exact singular solution:

$$
f_{1}(z ; t)=\sum_{j=\overline{1, N}} \delta\left(z-z_{j}(t)\right),
$$

where $z_{j}(t) \in T^{*}(\Lambda), j=\overline{1, N}$, - phase space coordinates in $T^{*}(\Lambda)^{N}$ of $N \in \mathbb{N}$ interacting particles in the domain $\Lambda \subset \mathbb{R}^{3}$. Specified above the Hamiltonicity problem and the 
existence of exact solutions to the Botzmann-Vlasov kinetic Equation (132) is deeply related to that of describing correlation functions $[19,20,38]$, suitably breaking the infinite Bogolubov chain $[10,19,34,38,52,53]$ of many-particle distribution functions. Namely, if to introduce manyparticle correlation functions $[19,20,38]$ for related Bogolubov distribution functions as

$$
\begin{aligned}
g_{1}\left(z_{1}\right)= & 0, g_{2}\left(z_{1}, z_{2}\right)=f_{2}\left(z_{1}, z_{2}\right)-f_{1}\left(z_{1}\right) f_{1}\left(z_{2}\right), \\
g_{3}\left(z_{1}, z_{2}, z_{3}\right)= & f_{3}\left(z_{1}, z_{2}, z_{3}\right)-f_{1}\left(z_{1}\right) f_{1}\left(z_{2}\right) f_{1}\left(z_{3}\right)-f_{1}\left(z_{1}\right) g_{2}\left(z_{2}, z_{3}\right)- \\
& -f_{1}\left(z_{2}\right) g_{2}\left(z_{3}, z_{1}\right)-f_{1}\left(z_{3}\right) g_{2}\left(z_{1}, z_{2}\right), \ldots,
\end{aligned}
$$

where $z_{j} \in T^{*}(\Lambda), j \in N$, then the Vlasov Equation (132) is obtained from the Bogolubov hierarchy at $n=1$ and $g_{2}\left(z_{1}, z_{2}\right)=0$ for all $z_{1}, z_{2} \in T^{*}(\Lambda)$.

As it was mentioned above, the constraint imposed on the infinite Bogolubov hierarchy is compatible with its Hamiltonicity. Yet in many practical cases this closedness procedure by means of imposing the conditions like

$$
g_{m+1}\left(z_{1}, z_{2}, . ., z_{m+1}\right)=0
$$

for all $z_{s} \in T^{*}(\Lambda), s=\overline{1, m+1}$ at some fixed $m \geq 2$ gives rise to some serious dynamical problems related with its mathematical correctness. Namely, if to close this way the infinite Bogolubov chain of kinetic equations on manyparticle distribution functions, one easily checks that the imposed constraint (135) does not persists in time subject to the evolution of the distribution functions $f_{j}\left(z_{1}, z_{2}, . ., z_{j}\right), z_{j} \in T^{*}(\Lambda), j=\overline{1, m}$. This means that these naively reduced kinetic equations are written down somehow incorrectly, as the reduced functional submanifold $M_{\mathcal{F}}^{(m)}:=\left\{\mathcal{F} \in M_{\mathcal{F}}: g_{m+1}=0\right\}$ should remain invariant in time. To dissolve this problem we are forced to consider the whole Bogolubov hierarchy of kinetic equations on multiparticle distribution functions as a Hamiltonian system on the functional manifold $M_{\mathcal{F}}$ and correctly reduce it on the constructed above functional submanifold $M_{\mathcal{F}}^{(m)} \subset M_{\mathcal{F}}$ via the classical Dirac type [7,21,22,24,59] procedure. The kinetic equations obtained this way by means of the reduced Lie-Poisson-Bogolubov structure will evidently differ from those naively obtained by means of the direct substitution of the imposed constraint (135) into the Bogolubov chain of kinetic equations, and in due course will conserve the functional submanifold $M_{\mathcal{F}}^{(m)} \subset M_{\mathcal{F}}$ invariant.

\subsection{The Invariant Reduction of the Bogolubov Distribution Functions Chain}

Consider the constructed before Hamiltonian functional $\mathcal{H}(\mathcal{F}) \in D\left(M_{\mathcal{F}}\right)$ (129)

$$
\mathcal{H}(\mathcal{F})=\int_{T^{*}(\Lambda)} d z_{1} \frac{p_{1}^{2}}{2 m} f_{1}\left(z_{1}\right)+\frac{1}{2} \int_{T^{*}(\Lambda)^{2}} d z_{1} d z_{2} V\left(x_{1}-x_{2}\right) f_{2}\left(z_{1}, z_{2}\right)
$$

and calculate the evolution of the distribution functions vector $\mathcal{F} \in M_{\mathcal{F}}$ under the simplest constraint (135) at $m=1$, that is

$$
g_{2}\left(z_{1}, z_{2}\right)=f_{2}\left(z_{1}, z_{2}\right)-f_{1}\left(z_{1}\right) f_{1}\left(z_{2}\right)=0
$$

for all $z_{1}, z_{2} \in T^{*}(\Lambda)$. To perform this reduction on $M_{\mathcal{F}}^{(1)} \subset M_{\mathcal{F}}$ we need $[7,24,59]$ to constraint the $\lambda$-extended Hamiltonian expression

$$
\mathcal{H}_{\lambda}(\mathcal{F}):=\mathcal{H}(\mathcal{F})+\frac{1}{2} \int_{T^{*}(\Lambda)^{2}} d z_{1} d z_{2} \lambda\left(z_{1}, z_{2}\right)\left[f_{2}\left(z_{1}, z_{2}\right)-f_{1}\left(z_{1}\right) f_{1}\left(z_{2}\right)\right]
$$


for some smooth function $\lambda \in D\left(T^{*}(\Lambda)^{2}\right)$ and next to determine it from the submanifold $M_{\mathcal{F}}^{(1)}$ invariance condition

$$
\begin{gathered}
\frac{\partial g_{2}\left(z_{1}, z_{2}\right)}{\partial t}=\left\{\left\{\mathcal{H}_{\lambda}(\mathcal{F}), g_{2}\left(z_{1}, z_{2}\right)\right\}\right\}= \\
=\frac{\partial f_{2}\left(z_{1}, z_{2}\right)}{\partial t}-\frac{\partial f_{1}\left(z_{1}\right)}{\partial t} f_{1}\left(z_{2}\right)-f_{1}\left(z_{1}\right) \frac{\partial f_{1}\left(z_{2}\right)}{\partial t}=0
\end{gathered}
$$

for all $z_{1}, z_{2} \in T^{*}(\Lambda)$ and $t \in \mathbb{R}$. To calculate effectively the condition (139) let us first calculate the evolutions for distribution functions $f_{1}$ and $f_{2} \in M_{\mathcal{F}}$ :

$$
\begin{aligned}
\frac{\partial f_{1}\left(z_{1}\right)}{\partial t}= & \left\{\left\{\mathcal{H}_{\lambda}(\mathcal{F}), f_{1}\left(z_{1}\right)\right\}\right\}=\left\{f_{1}\left(z_{1}\right), \frac{\delta \mathcal{H}_{\lambda}(\mathcal{F})}{\delta f_{1}\left(z_{1}\right)}\right\}^{(1)}+ \\
& +\int_{T^{*}(\Lambda)} d z_{2}\left\{f_{2}\left(z_{1}, z_{2}\right), \frac{\delta \mathcal{H}_{\lambda}(\mathcal{F})}{\delta f_{2}\left(z_{1}, z_{2}\right)}\right\}^{(1)},
\end{aligned}
$$

and

$$
\begin{gathered}
\quad \frac{\partial f_{2}\left(z_{1}, z_{2}\right)}{\partial t}=\left\{\left\{\mathcal{H}_{\lambda}(\mathcal{F}), f_{2}\left(z_{1}, z_{2}\right)\right\}\right\}=\left\{f_{2}\left(z_{1}, z_{2}\right), \frac{\delta \mathcal{H}_{\lambda}(\mathcal{F})}{\delta f_{1}\left(z_{1}\right)}+\frac{\delta \mathcal{H}_{\lambda}(\mathcal{F})}{\delta f_{1}\left(z_{2}\right)}\right\}^{(2)}+ \\
+\left\{f_{2}\left(z_{1}, z_{2}\right), \frac{\delta \mathcal{H}_{\lambda}(\mathcal{F})}{\delta f_{2}\left(z_{1}, z_{2}\right)}\right\}^{(2)}+\int_{T^{*}(\Lambda)} d z_{3}\left\{f_{3}\left(z_{1}, z_{2}, z_{3}\right), \frac{\delta \mathcal{H}_{\lambda}(\mathcal{F})}{\delta f_{2}\left(z_{1}, z_{3}\right)}+\frac{\delta \mathcal{H}_{\lambda}(\mathcal{F})}{\delta f_{2}\left(z_{2}, z_{3}\right)}\right\}^{(2)},
\end{gathered}
$$

which can be rewritten equivalently as follows:

$$
\begin{aligned}
\frac{\partial f_{1}\left(z_{1}\right)}{\partial t}= & -\left\langle\frac{\partial f_{1}\left(z_{1}\right)}{\partial p_{1}}\right| \int_{T^{*}(\Lambda)} d z_{2} \frac{\partial \lambda\left(z_{1}, z_{2}\right)}{\partial x_{1}} f_{1}\left(z_{2}\right)- \\
& -\left\langle\frac{p_{1}}{m}-\int_{T^{*}(\Lambda)} d z_{2} \frac{\partial \lambda\left(z_{1}, z_{2}\right)}{\partial p_{1}} f_{1}\left(z_{2}\right) \mid \frac{\partial f_{1}\left(z_{1}\right)}{\partial x_{1}}\right\rangle+ \\
& +\frac{1}{2} \int_{T^{*}(\Lambda)} d z_{2}\left\langle\frac{\partial}{\partial x_{1}}\left[V\left(x_{1}-x_{2}\right)+\lambda\left(z_{1}, z_{2}\right)\right] \mid \frac{\partial f_{2}\left(z_{1}, z_{2}\right)}{\partial p_{1}}\right\rangle- \\
& -\frac{1}{2} \int_{T^{*}(\Lambda)} d z_{2}\left\langle\frac{\partial \lambda\left(z_{1}, z_{2}\right)}{\partial p_{1}} \mid \frac{\partial f_{2}\left(z_{1}, z_{2}\right)}{\partial x_{1}}\right\rangle
\end{aligned}
$$

and

$$
\begin{aligned}
\frac{\partial f_{2}\left(z_{1}, z_{2}\right)}{\partial t}= & -\left\langle\frac{\partial f_{2}\left(z_{1}, z_{2}\right)}{\partial p_{1}} \mid \int_{T^{*}(\Lambda)} d z_{2} \frac{\partial \lambda\left(z_{1}, z_{2}\right)}{\partial x_{1}} f_{1}\left(z_{2}\right)\right\rangle- \\
& -\left\langle\frac{\partial f_{2}\left(z_{1}, z_{2}\right)}{\partial p_{2}} \mid \int_{T^{*}(\Lambda)} d z_{1} \frac{\partial \lambda\left(z_{1}, z_{2}\right)}{\partial x_{2}} f_{1}\left(z_{1}\right)\right\rangle- \\
& -\left\langle\frac{\partial f_{2}\left(z_{1}, z_{2}\right)}{\partial x_{1}} \mid \frac{p_{1}}{m}-\int_{T^{*}(\Lambda)} d z_{2} \frac{\partial \lambda\left(z_{1}, z_{2}\right)}{\partial p_{1}} f_{1}\left(z_{2}\right)\right\rangle- \\
& -\left\langle\frac{\partial f_{2}\left(z_{1}, z_{2}\right)}{\partial x_{2}} \mid \frac{p_{2}}{m}-\int_{T^{*}(\Lambda)} d z_{1} \frac{\partial \lambda\left(z_{1}, z_{2}\right)}{\partial p_{2}} f_{1}\left(z_{1}\right)\right\rangle+ \\
& +\frac{1}{2}\left\langle\frac{\partial f_{2}\left(z_{1}, z_{2}\right)}{\partial p_{1}} \mid \frac{\partial}{\partial x_{1}}\left[V\left(x_{1}-x_{2}\right)+\lambda\left(z_{1}, z_{2}\right)\right]\right\rangle+ \\
& +\frac{1}{2}\left\langle\frac{\partial f_{2}\left(z_{1}, z_{2}\right)}{\partial p_{2}} \mid \frac{\partial}{\partial x_{2}}\left[V\left(x_{1}-x_{2}\right)+\lambda\left(z_{1}, z_{2}\right)\right]\right\rangle-
\end{aligned}
$$




$$
\begin{aligned}
& -\frac{1}{2}\left\langle\frac{\partial f_{2}\left(z_{1}, z_{2}\right)}{\partial x_{1}} \mid \frac{\partial \lambda\left(z_{1}, z_{2}\right)}{\partial p_{1}}\right\rangle--\frac{1}{2}\left\langle\frac{\partial f_{2}\left(z_{1}, z_{2}\right)}{\partial x_{2}} \mid \frac{\partial \lambda\left(z_{1}, z_{2}\right)}{\partial p_{2}}\right\rangle+ \\
& +\frac{1}{2}\left\langle\int_{T^{*}(\Lambda)} d z_{3} \frac{\partial f_{3}\left(z_{1}, z_{2}, z_{3}\right)}{\partial p_{1}} \mid \frac{\partial}{\partial x_{1}}\left[V\left(x_{1}-x_{3}\right)+\lambda\left(z_{1}, z_{3}\right)\right]\right\rangle+ \\
& +\frac{1}{2}\left\langle\int_{T^{*}(\Lambda)} d z_{3} \frac{\partial f_{3}\left(z_{1}, z_{2}, z_{3}\right)}{\partial p_{2}} \mid \frac{\partial}{\partial x_{2}}\left[V\left(x_{2}-x_{3}\right)+\lambda\left(z_{2}, z_{3}\right)\right]\right\rangle- \\
& -\frac{1}{2}\left\langle\int_{T^{*}(\Lambda)} d z_{3} \frac{\partial f_{3}\left(z_{1}, z_{2}, z_{3}\right)}{\partial x_{1}} \mid \frac{\partial \lambda\left(z_{1}, z_{2}\right)}{\partial p_{1}}\right\rangle-\frac{1}{2}\left\langle\int_{T^{*}(\Lambda)} d z_{3} \frac{\partial f_{3}\left(z_{1}, z_{2}, z_{3}\right)}{\partial x_{2}} \mid \frac{\partial \lambda\left(z_{1}, z_{2}\right)}{\partial p_{2}}\right\rangle
\end{aligned}
$$

Having now substituted temporal derivatives (142) and (143) into the equality (139) in their explicit form, one obtains the following functional relationship:

$$
\begin{gathered}
\quad \frac{1}{2}\left\langle f_{1}\left(z_{2}\right) \frac{\partial f_{1}\left(z_{1}\right)}{\partial p_{1}}\right| \frac{\partial}{\partial x_{1}}\left(V\left(x_{1}-x_{2}\right)+\lambda\left(z_{1}, z_{2}\right)-\right. \\
\left.\left.-\int_{T^{*}(\Lambda)} d z_{3} f_{1}\left(z_{3}\right)\left[V\left(x_{1}-x_{3}\right)+\lambda\left(z_{1}, z_{3}\right)\right]\right)\right\rangle+ \\
+\frac{1}{2}\left\langle f_{1}\left(z_{1}\right) \frac{\partial f_{1}\left(z_{2}\right)}{\partial p_{2}}\right| \frac{\partial}{\partial x_{2}}\left(V\left(x_{2}-x_{1}\right)+\lambda\left(z_{2}, z_{1}\right)-\right. \\
\left.\left.-\int_{T^{*}(\Lambda)} d z_{3} f_{1}\left(z_{3}\right)\left[V\left(x_{2}-x_{3}\right)+\lambda\left(z_{2}, z_{3}\right)\right]\right)\right\rangle=0,
\end{gathered}
$$

which is satisfied if

$$
\lambda\left(z_{1}, z_{2}\right)=-V\left(x_{1}-x_{2}\right)
$$

for all $z_{1}, z_{2} \in T^{*}(\Lambda)$. Taking into account the result (145), one easily obtains from the Equation (142) the invariantly reduced on the submanifold $M_{\mathcal{F}}^{(1)} \subset M_{\mathcal{F}}$ kinetic equation on the one-particle distribution function:

$$
\frac{\partial f_{1}\left(z_{1}\right)}{\partial t}+\left\langle\frac{p_{1}}{m} \mid \frac{\partial f_{1}\left(z_{1}\right)}{\partial x_{1}}\right\rangle=\left\langle\frac{\partial f_{1}\left(z_{1}\right)}{\partial p_{1}} \mid \frac{\partial}{\partial x_{1}} \int_{T^{*}(\Lambda)} d z_{2} f_{1}\left(z_{2}\right) V\left(x_{1}-x_{2}\right)\right\rangle,
$$

which can be rewritten in the following compact form:

$$
\frac{\partial f_{1}\left(z_{1}\right)}{\partial t}=\left\{f_{1}\left(z_{1}\right), \frac{\delta \tilde{\mathcal{H}}(\mathcal{F})}{\delta f_{1}\left(z_{1}\right)}\right\}^{(1)}
$$

where we put, by definition,

$$
\tilde{\mathcal{H}}(\mathcal{F}):=\int_{T^{*}(\Lambda)} d z_{1} \frac{p_{1}^{2}}{2 m} f_{1}\left(z_{1}\right)+\frac{1}{2} \int_{T^{*}(\Lambda)^{2}} d z_{1} d z_{2} V\left(x_{1}-x_{2}\right) f_{1}\left(z_{1}\right) f_{1}\left(z_{2}\right) .
$$

The kinetic Equation (146) naturally coincides exactly with that obtained before from the naively reduced evolution equation

$$
\frac{\partial f_{1}\left(z_{1}\right)}{\partial t}=\left.\left\{\left\{\mathcal{H}(\mathcal{F}), f_{1}\left(z_{1}\right)\right\}\right\}\right|_{M_{\mathcal{F}}^{(1)}}
$$

on the submanifold $M_{\mathcal{F}}^{(1)} \subset M_{\mathcal{F}}$, as it is globally invariant [10,44] with respect to the classical Lie-Poisson-Bogolubov structure on $M_{\mathcal{F}}$. The same way as above one can explicitly construct the system of invariantly reduced kinetic equations

$$
\frac{\partial f_{1}\left(z_{1}\right)}{\partial t}=\left.\left\{\left\{\mathcal{H}(\mathcal{F}), f_{1}\left(z_{1}\right)\right\}\right\}\right|_{M_{\mathcal{F}}^{(2)}}, \frac{\partial f_{2}\left(z_{1}, z_{2}\right)}{\partial t}=\left.\left\{\left\{\mathcal{H}(\mathcal{F}), f_{2}\left(z_{1}, z_{2}\right)\right\}\right\}\right|_{M_{\mathcal{F}}^{(2)}}
$$

on the submanifold $M_{\mathcal{F}}^{(2)} \subset M_{\mathcal{F}}$, which already is not a priori globally invariant with respect to the Hamiltonian evolution flows on $M_{\mathcal{F}}$ and whose detail structure and analysis are postponed to another place. 


\section{Kinetic Theory of the Many-Particle Systems and Adsorption Phenomenon}

\subsection{Boltzmann-Bogolubov Equation, Surface Peculiarities, and Collision Integral}

Let us consider a one-component many-atoms dynamical system of the $N \in \mathbb{Z}_{+}$ particles, which are contained in the bounded volume $A \in \mathbb{R}^{3}$, where the density $N / V=$ $\rho>0$ is considered small enough. It allows us to use the developed before the theorey of the Bogolubov-Boltzmann kinetic equations for the dilute gases.

The Hamilton function of our dynamical system we write down as

$$
\mathcal{H}_{N}=\sum_{j=n}^{N} \frac{p_{j}^{2}}{2 m}+\frac{1}{2} \sum_{i \neq j=1}^{N} \mathrm{~V}\left(x_{i}-x_{j}\right)+\sum_{j=1}^{N} \mathrm{~V}_{0}\left(x_{j}\right),
$$

where $\mathrm{V}$ is interparticle interaction potential, $\mathrm{V}_{0}$ is a potential of the particle interaction with the domain $\Lambda \subset \mathbb{R}^{3}$ walls, that is with it surface $\partial \Lambda, z_{j}=\left(x_{j}, p_{j}\right) \in \mathbb{R}^{3} \times \mathbb{R}^{3}, j=\overline{1, N}$, and $N \in \mathbb{Z}_{+}$is a general number of particles. We assume that potential $V$ in (151) has Van der Waals nature, possesses the area of attraction as well as area of repulsion. The surface potential is very important, because it provide the condition of particle systems limitation in the fixed volume $\Lambda \subset \mathbb{R}^{3}$. It should be noted, that during analysis of particle interaction with the walls of volume $\Lambda \subset \mathbb{R}^{3}$, that is with the surface $\partial \Lambda$, the different non-potential models of the particle interaction with the surface are used.

Small particle density in the volume $\Lambda$ means that $\rho=N / V \ll a^{-3}$, where $a^{-3}$ is an effective volume of the one particle. For example, for the air under the normal conditions $\rho \approx 3 \cdot 10^{10} \mathrm{~cm}^{-3}, a^{-3} \approx 2 \cdot 10^{-4} \mathrm{~cm}$, that is $a^{-3} \approx 3 \cdot 10^{-4} \ll 1$. Under such conditions we can assume that gas particles in the volume $\Lambda \subset \mathbb{R}^{3}$ are moving most of the time freely, performing only frequent collision among each others.

Using the results of the previous section, namely the Equation (106) under condition (108), we can write down the following kinetic Bogolubov-Boltzmann Equation (76) for one-particle distribution function.

$$
\frac{\partial f(z ; t)}{\partial t}+\left\langle\frac{p}{m} \mid \nabla_{x} f(z ; t)\right\rangle=J(f)(z ; t)+J_{0}(f)(z ; t),
$$

where the collision integrals $J(f)$ and $J_{0}(f)$ in (152) are given by the following explicit expressions:

$$
\begin{aligned}
& J(f)(z ; t)=\int_{\mathbb{R}^{3} \times \mathbb{R}^{3}} d z^{\prime}\left\{f_{2}\left(z, z^{\prime}\right), \mathrm{V}\left(x-x^{\prime}\right)\right\}^{2}, \\
& J_{\sigma}(f)(z ; t)=\int_{\partial A} d \sigma \delta\left(x-x_{\sigma}\right)\left\{\chi\left(\left\langle p, n_{\sigma}\right\rangle\right) \int_{\mathbb{R}^{3}} d \rho^{\prime} \chi\left(\left\langle-p^{\prime} \mid n_{\sigma}\right\rangle\right) \times\right. \\
& \left.\times\left|\left\langle p^{\prime} \mid n_{\sigma}\right\rangle\right| K_{\sigma}\left(p, p^{\prime}\right) f\left(x, p^{\prime} ; t\right)-\chi\left(-\left\langle p \mid n_{\sigma}\right\rangle\right)\left|\left\langle p \mid n_{\sigma}\right\rangle\right| f(z ; t)\right\},
\end{aligned}
$$

where $d \sigma$ is infinitesimal element of the surface measure $\partial A$. The first item in (153) corresponds to interparticle collision in the volume $\Lambda \subset \mathbb{R}^{3}$, the second item to the collision with the surface of the container $\partial \Lambda, \chi(\alpha), \alpha \in \mathbb{R}$, is a standard Heaviside function, $(\chi(\alpha)=1, \alpha>0 ; \chi(\alpha)=0, \alpha \leqslant 0), K_{0}\left(p^{\prime}, p\right)$ is a kernel of the probabilistic distribution of the particles under the interaction with the surface $\partial \Lambda$. The point nature of the process is reflected by the Dirac $\delta$-function in (153). In the case of conservation on the number of particles in the container $A \subset \mathbb{R}^{3}$ the kernel $K_{\sigma}\left(p, p^{\prime}\right)$ should satisfy the natural condition:

$$
\int d \rho K_{\sigma}\left(p, p^{\prime}\right) \chi\left(\left\langle p \mid n_{\sigma}\right\rangle\right) \chi\left(-\left\langle p^{\prime} \mid n_{\sigma}\right\rangle\right)=1
$$

for all $p^{\prime} \in \mathbb{R}^{3}$ under condition $\left\langle p^{\prime} \mid n_{\sigma}\right\rangle<0$. The condition (154) means, that the walls of the container $\partial \Lambda$ are not adsorbing the particles and the probability of particle reflection from the wall $\partial \Lambda$ is equal to $1 ; n_{\sigma} \in \Lambda$ is a normal to the surface in the point $x_{\sigma} \in \partial \Lambda$. It is 
obvious, that under these limitations on the surface $\partial \Lambda$ the velocity with which in the given point the particles leave the surface, is equal the velocity of the collide with the surface under the given momentum of the scattering $p \in \mathbb{R}^{3},\left\langle p \mid n_{\sigma}\right\rangle>0$, that is

$$
\begin{aligned}
& \left|\left\langle p \mid n_{\sigma}\right\rangle\right| f\left(x_{\sigma}, p ; t\right) \chi\left(\left\langle p \mid n_{\sigma}\right\rangle\right)= \\
= & \int_{\mathbb{R}^{3}} d p^{\prime} \chi\left(-\left\langle p^{\prime} \mid n_{\sigma}\right\rangle\right) \chi\left(\left\langle p \mid n_{\sigma}\right\rangle\right) K_{\sigma}\left(p, p^{\prime}\right)\left|\left\langle p^{\prime} \mid n_{\sigma}\right\rangle\right| f\left(x_{\sigma}, p ; t\right)
\end{aligned}
$$

for all $t \in \mathbb{R}_{+}, x_{\sigma} \in \partial \Lambda$. Here, we will not concretize the explicit structure of the kernel $K_{\sigma}\left(p, p^{\prime}\right), p, p^{\prime} \in \mathbb{R}^{3}$, because of its strong dependency on the choice of interaction potential $\Phi_{0}$ in the Hamiltonian (151). It will be the object of a further more detail study.

\subsection{The Interactive Surface and Boundary Conditions}

As it was noted above, the distribution function $f(z ; t), z \in T^{*}(\Lambda), t \in \mathbb{R}_{+}$, as a solution of the Bogolubov-Boltzmann Equation (153), should be equal to zero in the area $\mathbb{R}^{3} \backslash \Lambda$, that is beyond the container $\Lambda \subset \mathbb{R}^{3}$ which contains the particles. This condition is realized by means of the following representation for the distribution function:

$$
f(z ; t)=\chi\left(x, x_{\sigma}\right) \tilde{f}(z ; t),
$$

where $z \in T^{*}(\Lambda), t \in \mathbb{R}_{+}$and $\chi\left(x, x_{\sigma}\right)$ is characteristic function of the surface $\partial \Lambda$, that is $\chi\left(x, x_{\sigma}\right)=1$ for $x \in \Lambda$ and $\chi\left(x, x_{\sigma}\right)=0$ for $x \in \mathbb{R}^{3} \backslash \Lambda$. Substituting expression (156) into (151) and (152) we can find that for all $x \in \Lambda, t \in \mathbb{R}_{+}$

$$
\begin{aligned}
& \frac{\partial \tilde{f}(z ; t)}{\partial t}+\left\langle\frac{p}{m} \mid \nabla_{x} \tilde{f}(z ; t)\right\rangle=J(\tilde{f})(z ; t), \quad\left\langle p \mid n_{\sigma}\right\rangle \tilde{f}\left(x_{\sigma}, p ; t\right)= \\
= & \int_{\mathbb{R}^{3}} d p^{\prime}\left|\left\langle p^{\prime} \mid n_{\sigma}\right\rangle\right| \chi\left(-\left\langle p^{\prime} \mid n_{\sigma}\right\rangle\right) K_{\sigma}\left(p, p^{\prime}\right) \tilde{f}\left(x_{\sigma}, p ; t\right),
\end{aligned}
$$

where $\left\langle p \mid n_{\sigma}\right\rangle>0, p \in \mathbb{R}^{3}$, and $\nabla_{x} \chi\left(x, x_{\sigma}\right)=\int_{\partial \Lambda} d \sigma n_{\sigma} \delta\left(x-x_{\sigma}\right)$. From the last equality we conclude, that for all $t \in \mathbb{R}_{+}$

$$
J(\tilde{f})\left(x_{\sigma}, p ; t\right)=0, \quad\left\langle p \mid n_{\sigma}\right\rangle>0,
$$

as a corollary of the dynamic equilibriumcondition (155). Thus, we obtained the necessary set of the boundary conditions for the distribution function $f(z ; t)=\tilde{f}(z ; t) \geqslant 0$ for $z \in T^{*}(\Lambda), t \in \mathbb{R}_{+}$, on the surface $\partial \Lambda$. These boundary conditions should be taken into account during solution kinetic Bogloubov-Boltzmann Equation (157) in the container $\Lambda \subset \mathbb{R}^{3}$.

\subsection{Adsorption Kinetics Equations}

Let us consider similar to the previous many-particle system in the volume $\Lambda \subset$ $\mathbb{R}^{3}$, where the part of the surface $\Gamma \subset \partial \Lambda$ has the adsorbing properties, that is the conditions $(154,155)$ are violated for $x_{\sigma} \in \Gamma$. It leads to accumulation of the adsorbed particles on the surface $\Gamma \subset \partial \Lambda$, which causes its dynamic equilibrium with the gas particles from the volume $\Lambda$ for $\ldots \rightarrow \infty$. In this case, the mechanism of the adsorption by the surface centers on $\Gamma$ needs to be additionally clarified. Let us represent the surface $\Gamma \subset \partial \Lambda$ as a discretely distributed set of the active adsorption centers with the potential energy of the activation $\mathcal{E}_{\sigma}=\frac{\left\langle p_{\sigma} \mid p_{\sigma}\right\rangle}{2 m}$ and the highlighted momenta $p_{\sigma} \in \mathbb{R}^{3}$, where $\left\langle p_{\sigma} \mid n_{\sigma}\right\rangle>0$, $\sigma \in \Gamma$. It means, that the surface $\Gamma$ one can imagine as a "spiky" lattice of the positively (inside $\Lambda$ ) oriented fixed impulse centers of adsorption. During particle "capturing" with the momentum $p=-p_{\sigma} \in \mathbb{R}^{3}$ by the surface $\Gamma \subset \partial \Lambda$ its total momentum $p=-p_{\sigma}+p_{\sigma}$ becomes zero and its corresponding "activation" energy $\varepsilon_{\sigma}=\frac{p_{\sigma}^{2}}{2 m}$. 
Thus, the basic information about adsorption process of the particles on the surface $\Gamma \subset \partial \Lambda$ is contained in the kernel of the collision integral $J_{\sigma}(f), \sigma \in \Gamma$, because on the other part of the surface adsorption does not occur. Taking this into account, we need to change the kinetic Equation (152) for this case, where, in particular, we need take into consideration the particle interactions in the volume $\Lambda$ with the adsorbed particles on the surface $\Gamma \subset \partial \Lambda$ and also the process of the adsorption center filling, which reduce the probability of its filling with time.

Let us write down the kinetic Equation (152) in the following general form

$$
\frac{\partial f(z ; t)}{\partial t}+\left\langle\frac{p}{m} \mid \nabla_{x} f(z ; t)\right\rangle=J(f)+J_{\Gamma}(f)+J_{\sigma \backslash \Gamma}(f),
$$

where for all $z \in \mathbb{R}^{3} \times \mathbb{R}^{3}, t \in \mathbb{R}$,

$$
\begin{aligned}
& J(f)(z ; t)=\int_{\mathbb{R}^{3} \times \mathbb{R}^{3}} d z^{\prime}\left\{f_{2}\left(z, z^{\prime}\right), \mathrm{V}\left(x-x^{\prime}\right)\right\}^{(2)}, \\
& J_{\Gamma}(f)(z ; t)=\int_{\partial \Lambda \backslash \Gamma} d \sigma \delta\left(x-x_{\sigma}\right)\left\{\chi\left(\left\langle p \mid n_{\sigma}\right\rangle\right) \int_{\mathbb{R}^{3}} d p^{\prime} \chi\left(-\left\langle p^{\prime} \mid n_{\sigma}\right\rangle\right) \times\right. \\
& \left.\times\left|\left\langle p^{\prime} \mid n_{\sigma}\right\rangle\right| K_{\sigma}\left(p, p^{\prime}\right) f\left(x, p^{\prime} ; t\right)-\chi\left(-\left\langle p \mid n_{\sigma}\right\rangle\right)\left|\left\langle p \mid n_{\sigma}\right\rangle\right| f(z ; t)\right\}, \\
& J_{\Gamma}(f)(z ; t)=\int_{\Gamma} d \sigma \delta\left(x-x_{\sigma}\right)\left\{\chi\left(\left\langle p \mid n_{\sigma}\right\rangle\right) \int_{\mathbb{R}^{3}} d p^{\prime} \chi\left(-\left\langle p^{\prime} \mid n_{\sigma}\right\rangle\right) \times\right. \\
& \left.\times\left|\left\langle p^{\prime} \mid n_{\sigma}\right\rangle\right| Q_{\sigma}\left(p, p^{\prime}\right) f\left(x, p^{\prime} ; t\right)-\chi\left(-\left\langle p \mid n_{\sigma}\right\rangle\right)\left|\left\langle p \mid n_{\sigma}\right\rangle\right| f(z ; t)\right\} .
\end{aligned}
$$

The kernel $Q_{\sigma}\left(p, p^{\prime}\right), p, p^{\prime} \in \mathbb{R}^{3}$, in (160) takes into account the nature of the gas particle collision in the volume $\Lambda \subset \mathbb{R}^{3}$ with the adsorbed centers on the surface $\Gamma \subset \partial \Lambda$. Taking into consideration that the representation (156) holds for the distribution function $f(z ; t), z \in T^{*}(\Lambda), t \in \mathbb{R}_{+}$, as a solution of the Equation (159), we get, that in the case $x \in \Lambda$ for all $t \in \mathbb{R}_{+}$

$$
\frac{\partial \tilde{f}(z ; t)}{\partial t}+\left\langle\frac{p}{m} \mid \nabla_{x} \tilde{f}(z ; t)\right\rangle=J(\tilde{f}) ;
$$

in the case $x_{\sigma} \in \partial \Lambda \backslash \Gamma, p \in \mathbb{R}^{3}$, and $\left\langle p \mid n_{\sigma}\right\rangle>0$

$$
\tilde{f}\left(x_{\sigma}, p ; t\right)\left|\left\langle p, n_{\sigma}\right\rangle\right|=\int_{\mathbb{R}^{3}} d p^{\prime} \chi\left(-\left\langle p^{\prime} \mid n_{\sigma}\right\rangle\right) K_{\sigma}\left(p, p^{\prime}\right)\left|\left\langle p^{\prime} \mid n_{\sigma}\right\rangle\right| \tilde{f}\left(x_{\sigma}, p ; t\right)
$$

in the case $x_{\sigma} \in \Gamma, p \in \mathbb{R}^{3},\left\langle p \mid n_{\sigma}\right\rangle>0$, the quantity

$$
-\tilde{f}\left(x_{\sigma}, p ; t\right)\left|\left\langle p \mid n_{\sigma}\right\rangle\right|+\int_{\mathbb{R}^{3}} d p^{\prime} \chi\left(-\left\langle p^{\prime} \mid n_{\sigma}\right\rangle\right) Q_{\sigma}^{+}\left(p, p^{\prime}\right)\left|\left\langle p^{\prime} \mid n_{\sigma}\right\rangle\right| \tilde{f}\left(x_{\sigma}, p ; t\right)=\frac{\partial \theta_{\sigma}^{+}(p ; t)}{\partial t}>0
$$

is a velocity of the particles runoff from the $\sigma$-part of the surface $\Gamma_{+} \subset \Gamma$, which is filled with the adsorbed particles; in the case $x_{\sigma} \in \Gamma_{-}, \Gamma \in \partial \Lambda$, and $\left\langle p \mid n_{\sigma}\right\rangle>0$ the quantity

$$
\tilde{f}\left(x_{\sigma}, p ; t\right)\left|\left\langle p \mid n_{\sigma}\right\rangle\right|-\int_{\mathbb{R}^{3}} d p^{\prime} \chi\left(-\left\langle p^{\prime} \mid n_{\sigma}\right\rangle\right) Q_{\sigma}^{-}\left(p, p^{\prime}\right)\left|\left\langle p^{\prime} \mid n_{\sigma}\right\rangle\right| \tilde{f}\left(x_{\sigma}, p ; t\right)=\frac{\partial \theta_{\sigma}^{-}(p ; t)}{\partial t}>0
$$

is a velocity of the adsorption of gas particles on the $\sigma$-center of the free part of the surface $\Gamma_{-} \subset \Gamma$. Here $\Gamma=\Gamma_{+} \cup \Gamma_{-}$, and we denoted the corresponding kernels in the collision integrals via $Q_{\sigma}^{+}$and $Q_{\sigma}^{-}$. It is obvious that kernel $Q_{\sigma}^{+}$corresponds to the collision integral 
of the gas particles on the similar particle but in the fixed form on the surface $\Gamma_{+}$, whereas the kernel $Q_{\sigma}^{-}$defined by nature of the adsorption centers on the part of the surface $\Gamma_{-} \subset \Gamma$.

We will use the obtained expressions (161)-(164) to find the integral velocity of the filling the adsorption centers on the surface $\Gamma \subset \partial \Lambda$. Namely, it is easy to observe, that the total number of the particles $\theta(t)>0$ on the surface $\Gamma \subset \partial \Lambda$ satisfies the following differential relationship

$$
\frac{\partial \theta(t)}{\partial t}=\int_{\Gamma_{-}} d \sigma \int_{\mathbb{R}^{3}} d p \chi\left(\left\langle p \mid n_{\sigma}\right\rangle\right) \frac{\partial \Theta_{\sigma}^{-}(p ; t)}{\partial t}-\int_{\Gamma_{+}} d \sigma \int_{\mathbb{R}^{3}} d p \chi\left(\left\langle p \mid n_{\sigma}\right\rangle\right) \frac{\partial \Theta_{\sigma}^{+}(p ; t)}{\partial t},
$$

which according with (161)-(164) is equivalent to the integral relationship in the form

$$
\frac{\partial \theta(t)}{\partial t}=\int_{\Gamma_{-}} d \sigma \int_{\mathbb{R}^{3}} d p \chi\left(\left\langle p \mid n_{\sigma}\right\rangle\right) T_{\sigma}^{-}(p ; \tilde{f})-\int_{\Gamma_{+}} d \sigma \int_{\mathbb{R}^{3}} d p \chi\left(\left\langle p \mid n_{\sigma}\right\rangle\right) T_{\sigma}^{+}(p ; \tilde{f}),
$$

where for all $p \in \mathbb{R}^{3},\left\langle p \mid n_{\sigma}\right\rangle>0$,

$$
\begin{aligned}
& T_{\sigma}^{\mp}(p, \tilde{f})= \pm \tilde{f}\left(x_{\sigma}, p ; t\right)\left|\left\langle p \mid n_{\sigma}\right\rangle\right| \mp \\
& \mp \int_{\mathbb{R}^{3}} d p^{\prime} \chi\left(-\left\langle p^{\prime} \mid n_{\sigma}\right\rangle\right) Q_{\sigma}^{\mp}\left(p, p^{\prime}\right)\left|\left\langle p^{\prime} \mid n_{\sigma}\right\rangle\right| \tilde{f}\left(x_{\sigma}, p ; t\right) .
\end{aligned}
$$

The obtained expression needs to be clarified, using the nature of adsorption centers distribution on the surface $\Gamma \in \partial \Lambda$ and fact, that the part of the surface $\Gamma_{+} \subset \Gamma$, which is filled by the adsorbed particles, contains them in the number of $\theta(t) \geqslant 0, t \in \mathbb{R}_{+}$. Thus, if the function of independent distribution $\omega_{\sigma} \in[0,1], \sigma \in \Gamma$, of the adsorption centers on the surface $\Gamma$ is given, then the integral Equation (166) can be rewritten as

$$
\frac{\partial \theta(t)}{\partial t}=\int_{\Gamma} d \sigma \omega_{\sigma} \tau_{\sigma}^{-}(\tilde{f})\left[1-\theta_{\sigma}(\tilde{f})\right]-\int_{\Gamma} d \sigma \omega_{\sigma} \tau_{\sigma}^{+}(\tilde{f}) \theta_{\sigma}(\tilde{f}),
$$

where we denoted

$$
\begin{aligned}
& \theta(t)=\int_{\Gamma} d \sigma \omega_{\sigma} \theta_{\sigma}(\tilde{f}), \quad \tau^{\mp}(\tilde{f})=\int_{\mathbb{R}^{3}} d p \chi\left(\left\langle p \mid n_{\sigma}\right\rangle\right) T_{\sigma}^{\mp}(p, \tilde{f}), \\
& \frac{\partial \theta_{\sigma}(\tilde{f})}{\partial t}=\left[1-\theta_{\sigma}(\tilde{f})\right] \tau_{\sigma}^{-}(\tilde{f})-\tau_{\sigma}^{+}(\tilde{f}) \theta_{\sigma},
\end{aligned}
$$

$\sigma \in \Gamma$. The system of the Equations (168) and (169) should be completed with one more Equation (157)

$$
\frac{\partial \tilde{f}(z ; t)}{\partial t}+\left\langle\frac{p}{m} \mid \nabla_{x} \tilde{f}(z ; t)\right\rangle=J(\tilde{f})(z ; t)
$$

for values $z \in \Lambda, t \in \mathbb{R}_{+}$, and the condition

$$
J_{\sigma}(\tilde{f})=0, \quad \sigma \in \partial \Lambda \backslash \Gamma
$$

on the distribution function $\tilde{f}(z ; t), z \in \Lambda$, in the volume $\Lambda$ in the vicinity of the element $\partial \Lambda$. The obtained system of the integral-differential Equations (168)-(171) is closed and gives the solution of the problem of construction the kinetic Bogolubov-Boltzmann equations in the bounded volume $\Lambda \subset \mathbb{R}^{3}$ with the surface peculiarities of it boundary $\partial \Lambda$.

In practice, when the area $\Gamma \subset \partial \Lambda$ of the container's $\Lambda$ boundary becomes adsorption active in the moment of time $t=0$ and when the distribution function $\tilde{f}(z ; t), z \in \Lambda, t \in \mathbb{R}_{+}$, for $t=0$ is equilibrium, that is maxwellian, the Equations (168) and (169) have analytical solution, which we do not write down here because of its being cumbersome. 


\subsection{Kernels of the Collision Integrals and Their Structure}

As it was mentioned above, during the study of the adsorption processes on the surface $\partial \Lambda$ of the volume $\Lambda$ of the many-particle dynamical system the kernels of the collision integrals of the particle's collision with particles with the surface and adsorbed on the surface particles play the main role. Let us consider the case of collision integral $J_{\sigma}^{+}(\tilde{f}), \sigma \in \Gamma_{+}$, that is collision of the particles from the volume $\Lambda \subset \mathbb{R}^{3}$ with the adsorbed particles on the the surface $\Gamma_{+} \subset \Gamma$. It is obvious that, in general case,

$$
J_{\sigma}(\tilde{f})=J_{\sigma}^{+}(\tilde{f})+J_{\sigma}^{-}(\tilde{f}),
$$

where $J_{\sigma}^{-}(\tilde{f}), \sigma \in \Gamma_{+}$, is a corresponding collision integral of the particles from the volume $\Lambda$ with the free from the adsorbed particles surface $\Gamma_{-} \subset \Gamma$, and for all $z \in \Lambda, t \in \mathbb{R}_{+}$

$$
\begin{aligned}
& J_{\sigma}^{ \pm}(\tilde{f})(z ; t)=\int_{\partial \Lambda} d \sigma \delta\left(x-x_{\sigma}\right)\left\{\chi\left(\left\langle p \mid n_{\sigma}\right\rangle\right) \int_{\mathbb{R}^{3}} d p^{\prime} \chi\left(-\left\langle p \mid n_{\sigma}\right\rangle\right) \times\right. \\
& \left.\times\left|\left\langle\frac{p^{\prime}}{m} \mid n_{\sigma}\right\rangle\right| Q_{\sigma}^{ \pm}\left(p^{\prime}, p\right) \tilde{f}\left(z_{\sigma}^{\prime} ; t\right)-\chi\left(\left\langle p \mid n_{\sigma}\right\rangle\right)\left|\left\langle\frac{p}{m} \mid n_{\sigma}\right\rangle\right| \tilde{f}(z ; t)\right\}
\end{aligned}
$$

Let us consider the collision integral $J_{\sigma}^{ \pm}(\tilde{f}), \sigma \in \Gamma_{+}$, based on the general expression for the collision integral $J_{\sigma}(\tilde{f})$ in the volume $\Lambda \subset \mathbb{R}^{3}$ under the condition, that the particle with the phase coordinate $z^{\prime} \in T^{*}(\Lambda)$ scatters on the adsorbed particle in the point $x=x_{\sigma} \in \Gamma_{+}$. Moreover, if the phase coordinate of the scattered particle on the surface is equal to $z=\left(x_{\sigma}, p\right) \in \Gamma_{+} \times \mathbb{R}^{3}$, then the collision momenta of the two particles satisfies the relationships $[2,6]$

$$
\begin{aligned}
& p=\tilde{p}^{\prime}=p^{\prime}-n_{\sigma}\left\langle n_{\sigma} \mid\left(p^{\prime}-\alpha\right)\right\rangle, \\
& \tilde{\alpha}=\alpha^{\prime}-n_{\sigma}\left\langle n_{\sigma} \mid\left(p^{\prime}-\alpha\right)\right\rangle,
\end{aligned}
$$

where $n_{\sigma} \in \mathbb{R}^{3}$ is a unit vector along the line of the centers of the interacting particles with the beginning in the point $x=x_{\sigma} \in \Gamma_{+}, p^{\prime} \in \mathbb{R}^{+},\left\langle n_{\sigma} \mid p^{\prime}\right\rangle<0$ is a momentum of the particle in the volume $\Lambda$ before the collision, $\tilde{p}^{\prime}=p \in \mathbb{R}^{3}$ is a momentum after the collision, $\alpha=p_{\sigma}$ is an own momentum of the adsorbed in the node $\sigma \in \Gamma_{+}$particle, which satisfies the natural condition of the particle not leaving the surface $\Gamma_{+}:\left\langle\tilde{p}_{\sigma}^{\prime}+p_{\sigma} \mid p_{\sigma}\right\rangle>0$, $\sigma \in \Gamma_{+}$. In the opposite case, when $\left\langle\tilde{p}_{\sigma}^{\prime}+p_{\sigma} \mid p_{\sigma}\right\rangle>0, \sigma \in \Gamma_{+}$, the adsorbed particle leaves the surface $\Gamma_{+} \subset \partial \Lambda$.

Let us write down the general expression for the collision integral $J_{\sigma}^{+}(\tilde{f}), \sigma \in \Gamma_{+}$:

$$
\begin{aligned}
& J_{\sigma}^{+}(\tilde{f})(z ; t)=\int_{T^{*}(\Lambda)} d z^{\prime}\left\{\tilde{f}_{\sigma}\left(z, z^{\prime} ; t\right), \mathrm{V}\left(x^{\prime}-x\right)\right\}^{(2)}= \\
= & \int_{T^{*}(\Lambda)} d z^{\prime}\left\langle\frac{p^{\prime}}{m} \mid \nabla_{x} \tilde{f}_{\sigma}\left(z, z^{\prime} ; t\right)\right\rangle \chi\left(-\left\langle p^{\prime} \mid n_{\sigma}\right\rangle\right)= \\
= & \int_{\Gamma_{+}} \delta\left(x-x_{\sigma}\right) \int_{\mathbb{R}^{3}} d p^{\prime} \chi\left(-\left\langle p^{\prime} \mid n_{\sigma}\right\rangle\right)\left\langle\frac{p^{\prime}}{m} \mid n_{\sigma}\right\rangle \times \\
& \times\left[\tilde{f}(z ; t) \tilde{f}_{\sigma}^{+}\left(p, p^{\prime}\right)-\tilde{f}\left(z_{\sigma}^{\prime} ; t\right) \tilde{f}_{\sigma}^{-}\left(p, p^{\prime}\right)\right] .
\end{aligned}
$$

Let us put

$$
\tilde{f}_{\sigma}\left(z, z^{\prime} ; t\right)=\tilde{f}\left(\tilde{z}^{\prime} ; t\right) \tilde{f}_{\sigma}\left(p, p^{\prime}\right) \delta\left(x-x_{\sigma}\right),
$$

as it was done in [2], where, by definition,

$$
\tilde{z}^{\prime}=\lim _{\tau \rightarrow \infty} S_{z}(-\tau) S_{z}^{(0)}(\tau) ; z^{\prime}=\lim _{\tau \rightarrow \infty} S_{z}(-\tau)\left(x^{\prime}+\frac{\tau p^{\prime}}{m}, p^{\prime}\right) ;
$$


$S_{z}(-\tau)$ is a generator of the canonical mappings group of the two-particle problem with the interaction potential, $S_{z}^{(0)}(\tau)$ is a corresponding generator of the canonical transformations group of the non-interaction two-particle problem, and

$$
\begin{aligned}
& \tilde{f}_{\sigma}^{+}\left(p, p^{\prime}\right)=\int_{\mathbb{R}^{3}} d \alpha \chi\left(\left\langle\alpha+p_{\sigma} \mid p_{\sigma}\right\rangle\right) \int d n_{\sigma} \chi\left(\left\langle n_{\sigma} \mid n_{\sigma}\right\rangle\right) \times \\
& \times \tilde{f}_{\sigma}\left(\alpha+n_{\sigma}\left\langle n_{\sigma} \mid p^{\prime}-\alpha\right\rangle\right) \delta\left(p+\vec{n}_{\sigma}\left\langle\vec{n}_{\sigma} \mid\left(p^{\prime}-\alpha\right)\right\rangle-p^{\prime}\right)
\end{aligned}
$$

is a probability scattering functions on the adsorbed node $\sigma \in \Gamma_{+}$after the particles collision,

$$
\begin{aligned}
& \tilde{f}_{\sigma}^{-}\left(p, p^{\prime}\right)=\int_{\mathbb{R}^{3}} d \alpha \chi\left(\left\langle\alpha+p_{\sigma} \mid p_{\sigma}\right\rangle\right) \int d n_{\sigma} \chi\left(\left\langle n_{\sigma} \mid n_{\sigma}\right\rangle\right) \times \\
& \times \tilde{f}_{\sigma}(\alpha) \delta\left(p+n_{\sigma}\left\langle n_{\sigma} \mid\left(p^{\prime}-\alpha\right)\right\rangle-p^{\prime}\right)
\end{aligned}
$$

is a probability scattering functions on the adsorbed node $\sigma \in \Gamma_{+}$before the particles collision. The function $\tilde{f}_{\sigma}(\alpha), \alpha \in \mathbb{R}^{3}$, in the most practical cases can be given as

$$
\tilde{f}_{\sigma}(\alpha)=C_{\sigma}^{+} \exp \left(-\frac{\langle\alpha \mid \alpha\rangle}{2 m_{\sigma}^{+} k T}\right)
$$

where $C_{\sigma}^{+}$is a normalizing constant of the density of the scattering centers states of the adsorbed particles on the surface $\Gamma_{+} \subset \partial \Lambda, m_{\sigma}^{+}$is their effective "surface particle"mass, and $\sigma \in \Gamma_{+}$. Thus, according to (176)-(179), the collision integral $J_{\sigma}^{+}(\tilde{f})(175)$ is given by the expression (173), where

$$
\begin{aligned}
& Q_{\sigma}^{+}(p, p)=C_{\sigma}^{+} \int_{\mathbb{R}^{3}} d \alpha \chi\left(\left\langle\alpha+p_{\sigma} \mid p_{\sigma}\right\rangle\right) \int d n_{\sigma} \chi\left(\left\langle n_{\sigma} \mid n_{\sigma}\right\rangle\right) \times \\
& \times \exp \left(-\frac{\langle\alpha \mid \alpha\rangle}{2 m_{\sigma}^{+} k T}\right) \delta\left(p+n_{\sigma}\left\langle n_{\sigma} \mid\left(p^{\prime}-\alpha\right)\right\rangle-p^{\prime}\right) .
\end{aligned}
$$

Here, one should also note that when the condition $\left\langle\alpha+n_{\sigma}\left\langle n_{\sigma} \mid\left(p^{\prime}-\alpha\right)\right\rangle+p_{\sigma} \mid p_{\sigma}\right\rangle<0$ for some pairs $\left(\alpha, p^{\prime}\right) \in \mathbb{R}^{3} \times \mathbb{R}^{3}$ is fulfilled, then after collision with the gas particle, which is adsorbed on the node $\sigma \in \Gamma_{+}$, the particle became free, that is the particle returns from the surface $\Gamma_{+}$to the volume $\Lambda \subset \mathbb{R}^{3}$. Thus, as it was noted before, the kernel $Q_{\sigma}^{+}\left(p, p^{\prime}\right)$, $p, p^{\prime} \in \mathbb{R}^{3}$ makes it possible to describe the process of the adsorbed particles releasing on the surface $\Gamma_{+}$. We do not stop here more on this in details and refer the reader to the explicit expressions (163) and (164).

By means of the similar to the described above method the problem of the calculation of the kernels of the scattering integrals on the surface $\Gamma_{-} \subset \Gamma$ and $\partial \Lambda \backslash \Gamma \subset \mathbb{R}^{3}$ can be solved. In particular, for the description of the elastic collision with the inertial adsorbing centers $\sigma \in \Gamma_{-}$we can get the following explicit expressions

$$
\begin{aligned}
& Q_{\sigma}^{-}\left(p, p^{\prime}\right)=C_{\sigma}^{-} \int_{\mathbb{R}^{3}} d \alpha \chi\left(\left\langle\alpha+p_{\sigma} \mid p_{\sigma}\right\rangle\right) \int d \tilde{n}_{\sigma} \chi\left(\left\langle\tilde{n}_{\sigma} \mid n_{\sigma}\right\rangle\right) \times \\
& \times \exp \left(-\frac{\langle\alpha \mid \alpha\rangle}{2 m_{\sigma}^{-} k T}\right) \delta\left(p+\tilde{n}_{\sigma}\left\langle n_{\sigma} \mid(p-\alpha)\right\rangle-p^{\prime}\right), \quad \sigma \in \Gamma_{-}, \\
& K_{\sigma}\left(p, p^{\prime}\right)=C_{\sigma} \int_{\mathbb{R}^{3}} d \alpha \chi\left(\left\langle\alpha+p_{\sigma} \mid p_{\sigma}\right\rangle\right) \int_{\sigma} d \tilde{n} \chi\left(\left\langle\tilde{n}_{\sigma} \mid n_{\sigma}\right\rangle\right) \times \\
& \times \exp \left(-\frac{\langle\alpha \mid \alpha\rangle}{2 m_{\sigma}^{-} k T}\right) \delta\left(p+\tilde{n}_{\sigma}\left\langle\tilde{n}_{\sigma} \mid\left(p^{\prime}-\alpha\right)\right\rangle-p^{\prime}\right), \quad \sigma \in \partial \Lambda \backslash \Gamma,
\end{aligned}
$$


where $C_{\sigma}^{-}, C_{\sigma} \in \mathbb{R}_{+}$are the corresponding normalizing constants, $m_{\sigma}, m_{\sigma}^{-}$are the inertial "masses" of the adsorbing centers on the surface $\partial \Lambda$. Thereby, we constructed concerted macroscopic theory of the kinetic processes in the many-particle dynamical systems in the bounded volume with taken into account adsorption phenomena, which are caused by the surface peculiarities.

Author Contributions: The main review idea and feature topics were conceived and suggested by L.I.I. and A.K.P., the first three Sections were prepared by Y.A.P. and V.H.S., the last sections were written jointly by A.K.P. and V.H.S. All authors have read and agreed to the published version of the manuscript.

Funding: This research received no external funding.

Institutional Review Board Statement: No humans and animals were involved into the research.

Informed Consent Statement: No humans and animals were involved into the research.

Data Availability Statement: There were used no supporting publicly archived datasets.

Acknowledgments: The deep Author's acknowledgements belong to the bright memory of recently passed away talented ukrainian physicist Lev Ivankiv with whom there were obtained most of the results of this work. Authors also would like to convey their warm thanks to Gerald A. Goldin for many discussions of the work and instrumental help in editing a manuscript during the XXVIII International Workshop on "Geometry in Physics", held on 30.06.-07.07.2019 in Białowieża, Poland. They also are cordially appreciated to Joel Lebowitz, Denis Blackmore and Nikolai N. Bogolubov (Jr.) for instructive discussions, useful comments and remarks on the work. A special author's appreciation belongs to Joel Lebowitz for the invitation to take part in the 121-st Statistical Mechanics Conference, held May 12-14, 2019 in the Rutgers University, New Brunswick, NJ, USA. Personal A.P.'s acknowledgement belongs to the Department of Physics, Mathematics and Computer Science of the Cracov University of Technology for a local research grant F-2/370/2018/DS.

Conflicts of Interest: The authors declare no conflict of interest.

\section{References}

1. Akhiezer, A.I.; Peletminsky, S.V. Methods of Statistical Physics; Pergamon Press: Oxford, UK, 2013.

2. Bogolyubov, N.N. Problems of Dynamical Theory in Statistical Physics; GITTL (State Publishing House for Technical and Theoretical Literature): Moscow-Leningrad, Russia, 1946. (In Russian)

3. Bogolubov, N.N.; Bogolubov, N.N., Jr. Introduction to Quantum Statistical Mechanics; Gordon and Breach: New York, NY, USA; London, UK, 1994.

4. Bogolubov, N.N., Jr.; Sadovnikov, B.I. Some Problems of Statistical Mechanics; Vyshaya Shkola Publisher: Moscow, Russia, 1975.

5. Bogolubov, N.N., Jr.; Sadovnikov, B.J.; Shumovsky, A.S. Mathematical Methods of Statistical Mechanical Model Systems; CRC: Boca Raton, FL, USA, 1984.

6. Petrina, D.Y.; Gerasimenko, V.I.; Malyshev, P.V. Mathematical Foundations of Classical Statistical Mechanics; CRC Press Publisher: Boca Raton, FL, USA, 2002.

7. Prykarpatsky, A.; Mykytyuk, I. Algebraic Integrability of Nonlinear Dynamical Systems on Manifolds: Classical and Quantum Aspects; Kluwer Academic Publishers: Dordrecht, The Netherlands, 1998.

8. Kac, M. Some Stochastic Problems in Physics and Mathematics, Colloquium Lectures in Pure and Applied Science; Magnolia Petroleum Co.: Dallas, TX, USA, 1956; Volume 2.

9. Chapman, S.; Cowling, T. Mathematical Theory of Non-Uniform Gases; Cambridge University Press: London, UK; New York, NY, USA, 1952.

10. Bogolubov, N.N., Jr.; Prykarpatsky, A.K. Quantum method of Bogolyubov generating functionals in statistical physics: Lie current algebra, its representations and functional equations. Sov. Part. Nucl. USA 1986, 17, 351-367.

11. Bogolubov, N.N., Jr.; Prykarpatsky, A.K. NN Bogolyubov's quantum method of generating functionals in statistical physics: The current Lie algebra, its representations and functional equations. Ukr. Mat. Zhurnal 1986, 38, 284-289.

12. Prykarpatsky, A.K.; Kaleniuk, P.I. Gibbs representations of current Lie algebra and quantum functional Bogoliubov equation. Doklady Acad. Nauk USSR 1988, 301, 871-876.

13. Goldin, G.A. Nonrelativistic current algebras as unitary representations of groups. J. Mathem. Phys. 1971, 12, 462-487. [CrossRef]

14. Goldin, G.A. Lectures on diffeomorphism groups in quantum physics. Contemporary Problems in mathematical Physics. In Proceedings of the Third International Workshop, Angra dos Reis, Brazil, 25-28 May 2004; World Scientific Publishing: Singapore, 2004; pp. 3-93 
15. Ivankiv, L.I.; Prykarpatski, A.K.; Samulyak, R.V. Non-Equilibrium Statistical Mechanics of Many-Particle Systems in Bounded Domain with Surface Peculiarities and Adsorption Phenomenon; Preprint N1-92; Institute for Applied Problems of Mechanics and Mathematics of NASU: Lviv, Ukraine, 1992.

16. Gibbon, J. Collisionless Boltzmann equations and integrable moment equations. Physica D 1981, 3, 502-511. [CrossRef]

17. Zakharov, V.E. The Benney equations and the quasiclassical approximation in the inverse scattering transform. Func. Anal. 1980, 14, 15-24. (In Russian)

18. Vlasov, A.A. Statistical Distribution Functions; Nauka Publisher: Moscow, Russia, 1966.

19. Balescu, M.R. Equilibrium and Non-Equilibrium Statistical Mechanics; Wiley: New York, NY, USA, 1975.

20. Zubarev, D.N. Nonequilibrium Statistical Thermodynamics; Consultants Bureau: New York, NY, USA, 1974.

21. Abraham, R.; Marsden, J. Foundations of Mechanics, 2nd ed.; Benjamin Cummings: New York, NY, USA,

22. Arnold, V.I. Mathematical Methods of Classical Mechanics; Springer: New York, NY, USA, 1978.

23. Lebedev, D.R.; Manin, Y.I. Benney's Long Wave Equations: Lax Representation and Conservation Laws. Zapiski Nauchnykh Seminarov LOMI.-1980-96; Boundary Value Problems of Mathematical Physics and Adjacent Function Theory Questions. pp. 169178. Available online: https:/ / www.opuscula.agh.edu.pl/vol27/2/art/opuscula_math_2715.pdf (accessed on 7 July 2021). (In Russian)

24. Blackmore, D.; Prykarpatsky, A.K.; Samoylenko, V.H. Nonlinear Dynamical Systems of Mathematical Physics: Spectral and DifferentialGeometrical Integrability Analysis; World Scientific Publ.: Hackensack, NJ, USA, 2011.

25. Kupershmidt, B. Hydrodynamical Poisson brackets and local Lie algebras. Phys. Lett. A 1987, 21, 167-174. [CrossRef]

26. Goldin, G.A.; Grodnik, J.; Powers, R.T.; Sharp, D. Nonrelativistic current algebra in the N/V limit. J. Math. Phys. 1974, 15, 88-100. [CrossRef]

27. Goldin, G.A.; Menikoff, R.; Sharp, F.H. Diffeomorphism groups, gauge groups, and quantum theory. Phys. Rev. Lett. 1983, 51, 2246-2249. [CrossRef]

28. Goldin, G.A.; Menikoff, R.; Sharp, F.H. Representations of a local current algebra in nonsimply connected space and the Aharonov-Bohm effect. J. Math. Phys. 1981, 22, 1664-1668. [CrossRef]

29. Goldin, G.A.; Sharp, D.H. Lie algebras of local currents and their representations. In Group Representations in Mathematics and Physics; Lecture Nootes in Physics; Springer: Berlin/Heidelberg, Germany, 1969; Volume 6, pp. 300-311.

30. Bratteli, O.; Robinson, D.W. Operator Algebras and Quantum Statistical Mechanics; Springer: Berlin/Heidelberg, Germany, 1997

31. Balakrishnan, A.V. Applied Functional Analysis; Springer: New York, NY, USA, 1981.

32. Bogolubov, N.N. Microscopic solutions of the Boltzmann-Enskog equation in kinetic theory for elastic balls. Theor. Math. Phys. 1975, 24, 804-807. [CrossRef]

33. Parthasarathy, K.R. Introduction to Probability and Measure; Hindustan Book Agency: New Delhi, India, 2005.

34. Bogolubov, N.N. Problems of Dynamical Theory in Statistical Physics; Geophysics Research Directorate, AF Cambridge Research Laboratories, Air Force Research Division, United States Air Force: Washington, DC, USA, 1960.

35. Bogolyubov, N.N., Jr.; Prykarpatsky, A.K. The Wigner quantized operator and N. N. Bogolyubov generating functional method in nonequilibrium statistical physics. Dokl. Akad. Nauk SSSR 1985, 285, 1365-1370.

36. Bazarov, I.P.; Gevorkian, E.V.; Nikolaev, P.N. Nonequilibrium Thermodynamics and Physical Kinetics; Moscow University Press: Moscow, Russia, 1989.

37. Bogolubov, N.N., Jr.; Prykarpatsky, A.K.; Samoilenko, V.H. Hamiltonian Structure of Hydrodynamical Benney Type Equations and Associated with Them Boltzmann-Vlasove Equations on Axis; Preprint of the Institute of Mathematics of NAS of Ukraine: Kiev, Ukraine, 1991; Volume N91.25, p. 43.

38. Libov, R. Introduction to the Theory of Kinetic Equations; Wiley: Hoboken, NJ, USA, 1969.

39. Boglolubov, N.N., Jr.; Brankov, J.G.; Zagrebnov, V.A.; Kurbatov, A.M.; Tonchev, N.S. Approximating Hamiltonian Method in Statistical Physics; Bulgarian Academy of Sciences Publ.: Sophia, Bulgaria, 1981.

40. Kozlov, V.V. Thermal Equilibrium in the Sense of Gibbs and Poincare; Inst. Komp'yut. Issled. Publisher: Izhevsk, Russia, 2002.

41. Kozlov, V.V. Gibbs Ensembles and Nonequilibrium Statistical Mechanics; Regulyarnaya i Khaoticheskaya Dinamika Publisher: Izhevsk, Russia, 2008.

42. The Vlasov kinetic equation, dynamics of continuum and turbulence. Regul. Chaotic Dyn. 2011, 16, 602-622. [CrossRef]

43. Lions, P.L.; Perthame, B. Propagation of Moments and Regularity for the 3-Dimensional Vlasov-Poisson System. Invent. Math. 1991, 105, 415-430. [CrossRef]

44. Marsden, J.E.; Morrison, P.J.; Weinstein, A. The Hamiltonian structure of the BBBGKY hierarchy equations. Contemp. Math. 1984, $28,115-124$.

45. Mikhaylov, A.I. The functional mechanics: Evolution of the moments of distribution function and the Poincare recurrence theorem. Vestn. Samar. Gos. Tekh. Univ. Fiz. Mat. Nauki 2011, 15, 124-133 [CrossRef]

46. Mikhaylov, A.I. The functional mechanics: Evolution of the moments of distribution function and the Poincare recurrence theorem. Adic Numbers Ultrametric Anal. Appl. 2011, 3, 205-211. [CrossRef]

47. Trushechkin, A.S. Microscopic solutions of kinetic equations and the irreversibility problem. Proc. Steklov Institute Math. Vol. 2014, 285, 251-274. [CrossRef]

48. Villani, C. A Review of Mathematical Topics in Collisional Kinetic Theory. In Handbook of Mathematical Fluid Dynamics; Friedlander, S., Serre, D., Eds.; Elsevier Science Publisher: Amsterdam, The Netherlands, 2002; Volume 1. 
49. Daletsky, Y.L.; Kadobyansky, R.M. The Poisson structures hierarchy and interacting ststems dynamics. Proceed. Ukr. Sci. 1994, 8 , 21-26.

50. Mandjavidze, J.; Sissakian, A. Generating functional method of N.N. Bogolubov and multiple production physics. arXiv 2000, arXiv:0003039v1.

51. Mendes, R.V. Current algebra, statistical mechanics and quantum models. arXiv 2017, arXiv:1711.03027v1.

52. Menikoff, R. Generating functionals determining representation of a nonrelativistic local current algebra in the $N / V$-limit. J. Math. Phys. 1974, 15, 1394-1408. [CrossRef]

53. Menikoff, R.; Sharp, D. Representation of a local current algebra: Their dynamical determination. J. Math. Phys. 1975, 16, 2341-2352. [CrossRef]

54. Volovich, I.V. Time irreversibility problem and functional formulation of classical mechanics. arXiv 2008, arXiv:0907.2445.

55. Weinstein, A. Sophus Lie and symplectic geometry. Expos. Math. 1983, 1, 95-96.

56. Weinstein, A. The local structure of Poisson manifolds. Differ. Geom. 1983, 18, 523-557. [CrossRef]

57. Hentosh, O.Y.; Balinsky, A.A.; Prykarpatski, A.K. The generalized centrally extended Lie algebraic structures and related integrable heavenly type equations. Carpathian Math. Publ. 2020, 12, 242-264. [CrossRef]

58. Bogolubov, N.N., Jr.; Prykarpatsky, A.K.; Samoilenko, V.H. Functional equations of N.N. Bogolubov and associated with them symplectic Lie-Poisson-Vlasov structure. Ukr. Math. J. 1986, 38, 747-778.

59. Dirac, P.A.M. The Principles of Quantum Mechanics, 2nd ed.; Clarendon Press: Oxford, UK, 1935. 